\title{
Transactive Energy Supported Economic Operation for Multi-Energy Complementary Microgrids
}

\author{
Yang, Zhao; Hu, Junjie; Ai, Xin; Wu, Jiechen; Yang, Guangya
}

Published in:

IEEE Transactions on Smart Grid

Link to article, DOI:

10.1109/TSG.2020.3009670

Publication date:

2021

Document Version

Peer reviewed version

Link back to DTU Orbit

Citation (APA):

Yang, Z., Hu, J., Ai, X., Wu, J., \& Yang, G. (2021). Transactive Energy Supported Economic Operation for MultiEnergy Complementary Microgrids. IEEE Transactions on Smart Grid, 12(1), 4 - 17.

https://doi.org/10.1109/TSG.2020.3009670

\section{General rights}

Copyright and moral rights for the publications made accessible in the public portal are retained by the authors and/or other copyright owners and it is a condition of accessing publications that users recognise and abide by the legal requirements associated with these rights.

- Users may download and print one copy of any publication from the public portal for the purpose of private study or research.

- You may not further distribute the material or use it for any profit-making activity or commercial gain

- You may freely distribute the URL identifying the publication in the public portal 


\title{
Transactive Energy Supported Economic Operation for Multi-Energy Complementary Microgrids
}

\author{
Zhao Yang, Junjie Hu, Member, IEEE, Xin Ai, Member, IEEE, Jiechen Wu, Guangya Yang, Senior \\ Member, IEEE
}

\begin{abstract}
Multi-energy complementary microgrids (MECMs) provide an important means to accommodate renewable energy sources due to their abundant adjustable resources and flexible operation modes. However, limited capacity and controllability are the main obstacles that prevent MECMs from participating in the market. In this study, we develop a transactive energy (TE) mechanism-supported energy sharing strategy to coordinate interconnected MECMs in a regional integrated energy system (RIES), where the uncertainty of renewable energy and loads is taken into account via stochastic programming. An RIES operator is introduced to trade with the utility grid as an intermediate player between the electricity market and MECMs. For the TE mechanism, we employ alternating direction method of multipliers (ADMM) algorithm to achieve distributed optimization of energy sharing, which is based on the average of the shared energy residual over all MECMs. A clear economic interpretation exists in the method, wherein shared electrical and thermal energy prices can be obtained. Case studies demonstrate the effectiveness of the multi-energy sharing scheme considering integrated demand response (IDR). Moreover, the distributed algorithm can be implemented and converge easily while respecting MECMs' individual benefits and private information.

Index Terms-Alternating direction method of multipliers (ADMM), energy sharing, integrate demand response (IDR), multi-energy complementary microgrids (MECMs), transactive energy (TE).

\section{NOMENCLATURE}

$\begin{array}{ll}\text { Acronym } & \\ \text { TE } & \text { Transactive Energy } \\ \text { MECM } & \text { Multi-energy complementary microgrid } \\ \text { RIES } & \text { Regional integrated energy system } \\ \text { CCHP } & \text { Combined cooling, heat and power } \\ \text { DERs } & \text { Distributed energy resources } \\ \text { IDR } & \text { Integrated demand response } \\ \text { MT } & \text { Micro turbine } \\ \text { HX } & \text { Heat exchanger } \\ \text { AC } & \text { Adsorption chillers } \\ \text { TCLs } & \text { Thermostatically controlled loads } \\ \text { WH } & \text { Water heater }\end{array}$
\end{abstract}

This work is supported in part by the National Natural Science Foundation of China (51877078) and partly by the Beijing Natural Science Foundation (3182037), China.

Z. Yang, J. Hu, X. Ai and J. Wu are with the State Key Laboratory of Alternate Electrical Power System With Renewable Energy Sources (North China Electric Power University), Beijing, 102206, China (e-mail: joyz0413@163.com; junjiehu@ncepu.edu.cn; aixin@ncepu.edu.cn; wjcncepu@foxmail.com) (corresponding author: J. Hu)

G. Yang is with the Center for the Electric Power and Energy, Technical University of Denmark, Lyngby, Denmark (e-mail: gyy@elektro.dtu.dk).

\begin{tabular}{|c|c|}
\hline HVAC & Heating, ventilation air conditioning \\
\hline HP & Heat pump \\
\hline ESS & Energy storage system \\
\hline EV & Electric vehicle \\
\hline PV & photovoltaic \\
\hline \multicolumn{2}{|c|}{ Set and Indices } \\
\hline $\mathrm{Nb}$ & Set of MECMs participating in the energy sharing \\
\hline$N b_{w h}$ & Set of water heater in MECM $b$ \\
\hline$N b_{m}$ & Set of HVAC system in MECM $b$ \\
\hline$N b_{v}$ & Set of EVs in MECM $b$ \\
\hline Ns & Set of representative scenarios \\
\hline$n$ & Index of water heater \\
\hline$m$ & Index of HVAC system \\
\hline$b$ & Index of MECM \\
\hline$t$ & Index of scheduling time period \\
\hline$s$ & Index of scenario \\
\hline$i$ & Row index of auxiliary variable matrix \\
\hline$k$ & Index of iteration round \\
\hline \multicolumn{2}{|l|}{ Parameter } \\
\hline$N T$ & Number of scheduling periods \\
\hline$\Delta t$ & Duration of a time slot \\
\hline$P_{\max }^{\mathrm{mt}}$ & Upper bounds of electrical power of MT \\
\hline$H_{\max }^{\mathrm{mt}}$ & Upper bounds of heat power of MT \\
\hline$\eta^{\text {mte }}$ & Power generation efficiency of MT \\
\hline$\eta^{\text {loss }}$ & Heat loss coefficient of MT \\
\hline$C_{o p h}$ & Heating coefficient \\
\hline$L^{\text {hvng }}$ & Low calorific value of natural gas \\
\hline$H_{\max }^{\mathrm{hx}}$ & Maximum input heat power of heat exchanger \\
\hline$H_{\max }^{\mathrm{ac}}$ & Maximum input heat power of adsorption chillers \\
\hline$c_{\mathrm{wh}}, r_{\mathrm{wh}}$ & $\begin{array}{l}\text { Thermal capacitance and thermal resistance of hot } \\
\text { tank }\end{array}$ \\
\hline$\theta_{t}^{\mathrm{wh}}, \theta_{t}^{\mathrm{amb}}$ & Hot water temperature and ambient temperature \\
\hline$q_{n, t}^{\mathrm{wh}}$ & Heat input from water heater \\
\hline$q_{n, t}^{\text {hwd }}$ & Demand heat power for hot water in $\operatorname{tank} n$ \\
\hline$V_{n, t}^{\mathrm{hwd}}$ & Hot water demand volume in tank $n$ \\
\hline$\theta_{\mathrm{wh}}^{\min }, \theta_{\mathrm{wh}}^{\max }$ & $\begin{array}{l}\text { Minimum and maximum temperature limits of hot } \\
\text { water }\end{array}$ \\
\hline$\eta_{b}^{\mathrm{hx}}$ & Efficiency of heat exchanger. \\
\hline$\theta_{m, t}^{\mathrm{a}}, \theta_{m, t}^{\mathrm{w}}$ & Indoor temperature of HVAC and wall temperature \\
\hline$Q_{m, t}^{\text {hvac }}$ & Cooling power of HVAC $m$ \\
\hline $\mathrm{A}_{m}, \mathrm{~B}_{m}, C_{m}$ & $\begin{array}{l}\text { Coefficients matrices of second-order state-space } \\
\text { HVAC model and disturbance vector }\end{array}$ \\
\hline$\delta_{m, t}$ & Disturbance vector \\
\hline$\theta_{t}^{\min }, \theta_{t}^{\max }$ & Maximum and minimum indoor temperature \\
\hline$Q_{b, t}$ & Total cooling power consumption in MECM $b$ \\
\hline
\end{tabular}




$\begin{array}{ll}Q_{b, t}^{\text {ac }} & \text { Cooling power output of adsorption chillers } \\ Q_{b, t}^{\text {hp }} & \text { Cooling power output of heat pump } \\ \mathrm{CoP}^{\text {ac }}, \mathrm{CoP}^{\mathrm{hp}} & \begin{array}{l}\text { Coefficient of performance of adsorption chillers } \\ \text { and heat pump, respectively }\end{array} \\ C^{\text {bat }} & \begin{array}{l}\text { Total investment on the battery } \\ \text { Charge/discharge cycle number during the life } \\ \text { cycle, battery capacity, depth of discharge of the }\end{array} \\ L^{\mathrm{c}}, E, d^{\mathrm{DOD}} & \text { battery } \\ P_{\max }^{\text {ch, }}, P_{\max }^{\mathrm{dsh}} & \text { Maximum charge and discharge power of EV/ESS } \\ \mathrm{SoC}_{i, t}^{\mathrm{EVIESS}} & \text { State of charge of EV/ESS } i \text { at slot } t \\ \mathrm{SoC}_{i, 0}^{\mathrm{ESS}}, \operatorname{SoC}_{i, T}^{\mathrm{ESS}} & \begin{array}{l}\text { SoC of ESS } i \text { at the beginning and end of the time } \\ \text { horizon }\end{array}\end{array}$

\section{INTRODUCTION}

$\mathrm{P}$ OWER system is experiencing a fundamental transition with the high penetration of distributed energy resources (DERs) in end users. Traditional passive consumers have converted to proactive prosumers [1]. With local supplementation, energy sharing provides an effective management approach that brings additional value [2]-[4], including sufficient utilization of source flexibility, reduction of transmission energy loss, improved acceptance of DERs, etc. The authors of [5], [6] propose an energy management strategy to coordinate energy sharing among prosumers by a centralized agent. However, centralized management requires detailed data of prosumers to yield the optimal scheme from the global perspective, which results in computation and communication bottlenecks as well as privacy violations [7].

To address this, it is crucial to develop an adaptive energy sharing framework while preserving prosumers' autonomous decisions. Transactive energy (TE) is such a scalable and distributed approach that integrates economic and control mechanisms to achieve equilibrium using value as a key operational parameter [8]. Distributed energy management strategies based on dual price (e.g., dual decomposition [9], alternating direction method of multipliers, ADMM [10]-[12]) provide a favorable framework to model the TE-supported energy sharing problem [13], where the iterations can be interpreted as a price-directed optimization mechanism [10]. In [9], a dual decomposition algorithm method is designed to obtain distributed energy sharing strategy, which explains the algorithm iterations in terms of "supply-demand balance". Compared with dual decomposition algorithms, the ADMM has the advantages of easy distributed implementation, scalability, and good convergence properties [14]. In [10]-[12], ADMM-based optimization is developed for energy sharing management, and economic dispatch aiming to minimize the total cost is converted to several prosumers' individual programs and aggregators' local programs. Using the ADMM algorithm, prosumers' optimization problems can be solved independently, and private information is protected to a large extent. However, centralized optimization is still required to coordinate all the prosumers, and the coupled constraint about the shared energy balance remains to be solved by the aggregator. This approach is unsuitable if the scale of prosumers expands because of the computing pressure on the aggregator and the limited scalability. Therefore, a novel ADMM algorithm based on the average of supply-demand imbalance rather than the whole residual is supposed in [2], [15], [16]. The author of [2] derives that the dual prices of shared energy between one microgrid and any other microgrid can be uniform, and the average value of the energy imbalance is used to simplify the distributed optimization. In [16], energy sharing is coordinated by a supervisory third party by considering the average energy residual over all prosumers. The oscillations are smoothed, and the method converges more easily.

Nevertheless, the energy sharing in the above studies is limited to the level of electric energy. With the popularization of integrated energy systems (IESs), the practical energy demands of users might include multiple energy types other than electricity, such as combined cooling, heat and power (CCHP) systems [17], which suggests more complex integrated demand models. Multi-energy prosumers have gradually become the mainstream research direction [18], [19]. In an integrated energy system, multi-energy prosumers do not only exchange energy through a physical connection of power grid. The heating network also offers substantial potential for heat storage and requires no additional investment; if appropriately controlled, the flexibility can reveal strategic opportunities for supply and demand in the IES [19]-[21]. The authors of [19] propose coordinated operational strategies among regional multi-energy prosumers through a ring heat network. To motivate the IES to take advantage of the flexibility, a heat storage pipeline is used to internally trade heat energy in the community with a CCHP system in [21], in which single electric energy sharing is extended to multi-energy sharing. In [22], multi-energy sharing scheduling of an integrated energy microgrid cluster is modeled as a Stackelberg game. For heat-electricity integrated energy systems, a hybrid energy sharing scheme is derived in a distributed way in [23]-[24].

However, the aforementioned research sets the heat load as a fixed load [19], [21], [22] or punishes the difficulty caused by a deviation from the set load [23], [24], regardless of the actual energy demand of users. Note that thermostatically controlled loads (TCLs) account for a large proportion of residential heat loads, whose energy consumption is flexible due to thermal inertia characteristics [25]. With the assistance of an integrated demand response (IDR) strategy, such as price guidance [25] or multi-energy complementarity [17], energy sharing scheduling can leverage the flexibilities available from multiple prosumers more effectively.

Taken together with the mentioned works, this study aims to make essential improvements consisting of fully exploring and utilizing the flexibility of MECM systems through IDR and multi-energy sharing, and providing an efficient distributed optimization for multi-energy sharing problems. With these motivations, we investigate a multi-energy sharing strategy for a typical multi-energy prosumer, multi-energy complementary microgrid (MECM), considering uncertainty factors. The main contributions of this work are summarized as follows:

1) Single electrical energy sharing scheme is extended to multi-energy sharing of electrical and thermal energy in an 
RIES while considering IDR at the MECM level. Under this scheme, local self-consumption of interconnected multi-energy prosumers can be accomplished while improving the economy and flexibility of MECMs system.

2) Distributed optimization is formulated for the multi-energy sharing problem to implement the self-organization of MECMs, which considers the uncertainty of photovoltaic and load demand via scenario-based stochastic programming.

3) On the basis of a TE mechanism, a multi-energy sharing balance can be achieved through economic signals. We interpret the ADMM algorithm from an economic perspective and obtain market-clearing prices of shared electrical and thermal energy, which provides a price scheme for multi-energy sharing of thermal-electric coupling systems. Additionally, the mechanism integrates multiple scenarios to account for the impact of randomness.

The remainder of this paper is organized as follows. Section II introduces a distributed multi-energy sharing framework for multiple MECMs from the perspective of energy and information interaction, respectively. Models of key components of an MECM are presented in Section III. In Section IV, MECMs scenario-based stochastic optimization model is established and decoupled through ADMM, and economic interpretation of ADMM is given. Section V discusses the case studies of multi-energy sharing as well as the paper concludes with Section VI.

\section{Multi-EnERGy Sharing SCHEME}

\section{A. System operational framework}

In this section, we propose a TE-supported multi-energy sharing framework for multiple interconnected MECMs.

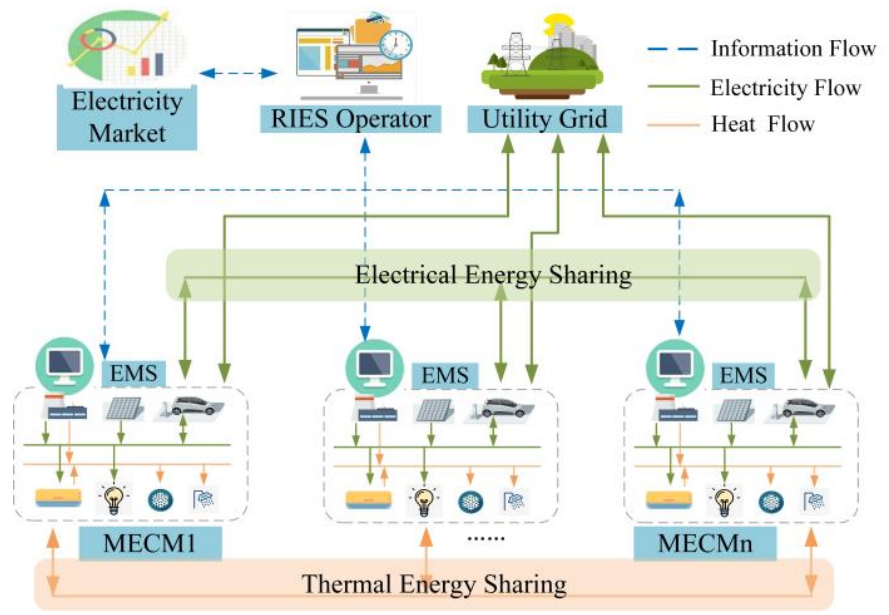

Fig. 1. Operational framework for the energy-sharing distribution system

In Fig. 1, the MECM contains a CCHP system, PV and demand-side management (DSM) resources, such as EVs/ESSs and TCLs, which can be regarded as prosumers for both electrical and thermal energy. Surplus energy from the MECM can be shared through the local energy sharing network. Considering that the long-distance transmission of thermal energy will cause energy loss, in this work, thermal energy is set to be shared only among local neighboring MECMs. After internal energy sharing is performed, the remaining electricity deficit or surplus can be traded with the utility at the market price.

As mentioned previously, centralized scheduling of energy sharing is considered challenging given the communication pressure and privacy leaks. To this end, a distributed framework for energy sharing is developed in our work. In terms of distributed energy management, the global optimization problem is decomposed into MECMs' individual subproblems. Each MECM energy management system (EMS) determines its own optimization scheme locally, and then the information including expected shared electrical and thermal power, power interacting with the utility grid, is submitted to the regional IES (RIES) operator. As the intermediate player between the electricity market and MECMs, the RIES operator manages the energy sharing process and participates in the electricity market on behalf of MECMs. Based on the expected shared energy from each MECM, the RIES operator coordinates energy sharing toward supply-demand balance and publishes the guiding signal to each MECM-EMS. Meanwhile, each MECM updates its optimal scheduling accordingly. The iteration process is described in Section IV. The energy that MECMs interact with the grid will be reported to the entire market through the RIES operator, which is used as the basis for dispatching in the market.

\section{Models Of Key COMPONENTS In AN MECM}

\section{A. CCHP System}

The key device of a CCHP system is micro turbine (MT). CCHP systems convert natural gas into electricity, and then the waste heat from the MT is used to provide heat using heat exchanger (HX) or produce cooling energy by adsorption chillers (AC).

$$
P_{b, t}^{\mathrm{mt}}=F_{b, t}^{\mathrm{gas}} \eta^{\mathrm{mte}} L^{\mathrm{hng}} / \Delta t
$$

The relationship between the electric power and heat of an MT [24] is described as follows:

$$
H_{b, t}^{\mathrm{mt}}=P_{b, t}^{\mathrm{mt}} \frac{\left(1-\eta^{\mathrm{mte}}-\eta^{\text {loss }}\right) C_{o p h}}{\eta^{\mathrm{mte}}}
$$

where $\eta^{\text {mte }}$ is the generation efficiency of the MT, which is assumed to be constant in this paper.

The power range of CCHP systems is written as follows:

$$
\begin{aligned}
& 0 \leq P_{b, t}^{\mathrm{mt}} \leq P_{\max }^{\mathrm{mt}} \\
& 0 \leq H_{b, t}^{\mathrm{mt}} \leq H_{\max }^{\mathrm{mt}} \\
& 0 \leq H_{b, t}^{\mathrm{hx}} \leq H_{\max }^{\mathrm{hx}} \\
& 0 \leq H_{b, t}^{\mathrm{ac}} \leq H_{\max }^{\mathrm{ac}}
\end{aligned}
$$

\section{B. Model of TCLs}

\section{1) Water heater model}

In this work, a resistance-capacitance circuit model [25] is employed to describe the temperature dynamics of a water heater as follows: 


$$
c_{\mathrm{wh}} \frac{\mathrm{d} \theta_{n, t}^{\mathrm{wh}}}{\mathrm{d} t}=q_{n, t}^{\mathrm{wh}}-q_{n, t}^{\mathrm{hwd}}+\frac{\theta_{t}^{\mathrm{amb}}-\theta_{n, t}^{\mathrm{wh}}}{r_{\mathrm{wh}}}
$$

where $q_{n, t}^{\text {hwd }}$ represents the demand heat power for hot water in tank $n$, which is given by

$$
q_{n, t}^{\mathrm{hwd}} \Delta t=C_{\mathrm{p}} \rho V_{n, t}^{\mathrm{hwd}}\left(\theta_{n, t}^{\mathrm{wh}}-\theta_{t}^{\mathrm{amb}}\right)
$$

The relationship among $\theta_{k, t}^{\mathrm{wh}}, q_{k, t}^{\mathrm{wh}}$ and $\theta_{t}^{\mathrm{amb}}$ can be expressed in the following discrete form:

$$
\theta_{n, t+1}^{\mathrm{wh}}=a_{0} \theta_{n, t}^{\mathrm{wh}}+b_{0} q_{n, t}^{\mathrm{wh}}+c_{0} \theta_{t}^{\mathrm{amb}}
$$

where $\quad a_{0}=1-\frac{\Delta t}{r_{\mathrm{wh}} c_{\mathrm{wh}}}-\frac{C^{p} \rho V_{n, t}^{\mathrm{hwd}}}{c_{\mathrm{wh}}} \quad, \quad b_{0}=\frac{\Delta t}{c_{\mathrm{wh}}} \quad$ and $c_{0}=\frac{\Delta t}{r_{\mathrm{wh}} c_{\mathrm{wh}}}+\frac{C_{\mathrm{p}} \rho V_{n, t}^{\mathrm{hwd}}}{c_{\mathrm{wh}}}$ in detail.

In addition, the hot water temperature must be kept within an acceptable range to satisfy the water temperature requirement of occupants.

$$
\theta_{\mathrm{wh}}^{\min } \leq \theta_{n, t}^{\mathrm{wh}} \leq \theta_{\mathrm{wh}}^{\max }
$$

In MECM $b$, the heat power of the $N b_{w h}$ water heater is supplied by a heat exchanger, as given by

$$
\eta_{b}^{\mathrm{hx}} H_{b, t}^{\mathrm{hx}}=\sum_{n=1}^{N b_{\mathrm{whh}}} q_{n, t}^{\mathrm{wh}}
$$

\section{2) HVAC model}

In accordance with the second-order state-space model of the HVAC system proposed in [26], the thermal dynamic model is described as follows:

$$
\begin{gathered}
\theta_{m, t+1}=\mathrm{A}_{m} \theta_{m, t}+\mathrm{B}_{m} \mathrm{u}_{m, t}+\delta_{m, t} \\
\theta_{m, t}^{\mathrm{a}}=C_{m} \theta_{m, t}
\end{gathered}
$$

where $\theta_{m, t}=\left[\begin{array}{ll}\theta_{m, t}^{\mathrm{a}} & \theta_{m, t}^{\mathrm{w}}\end{array}\right]^{\mathrm{T}}$ is the state vector, $\theta_{m, t}^{\mathrm{a}}$ and $\theta_{m, t}^{\mathrm{w}}$ represent the indoor temperature and wall temperature, respectively, $\mathbf{u}_{m, t}$ is the input control vector with $\mathrm{u}_{m, t}=\left[\mathrm{Q}_{m, t}^{\text {hvac }} 0\right]^{\mathrm{T}}$, and $\mathrm{Q}_{m, t}$ is the cooling power of room $m$. $\delta_{m, t}$ is the disturbance vector, including the impact of ambient temperature and solar irradiation. The coefficients of matrices $\mathrm{A}_{m}$ and $\mathrm{B}_{m}$ can be calculated based on the effective wall area, window area and heat transfer rate, $C_{m}=\left[\begin{array}{ll}1 & 0\end{array}\right]$.

Similarly, the temperature limit is enforced by

$$
\theta_{t}^{\min } \leq \theta_{m, t}^{a} \leq \theta_{t}^{\max }
$$

In MECM $b$, the total cooling power consumption of $N b_{m}$ HVACs is expressed as

$$
Q_{b, t}=\sum_{m=1}^{N b_{m}} Q_{m, t}^{\mathrm{hvac}}
$$

The occupant's cooling demand is fulfilled by an electric heat pump and adsorption chillers consuming the waste heat delivered from the micro turbine, which are displayed as follows:

$$
\begin{aligned}
& Q_{b, t}=Q_{b, t}^{\mathrm{ac}}+Q_{b, t}^{\mathrm{hp}} \\
& Q_{b, t}^{\mathrm{ac}}=\operatorname{CoP}^{\mathrm{ac}} H_{b, t}^{\mathrm{ac}}
\end{aligned}
$$

$$
Q_{b, t}^{\mathrm{hp}}=\mathrm{CoP}^{\mathrm{hp}} P_{b, t}^{\mathrm{hp}}
$$

\section{Electric Vehicles and Energy Storage System}

The charging and discharging processes of electric vehicles (EVs) [27]-[28] and energy storage system (ESS) [2] are introduced in the model. For simplicity, we use the same representation for ESS and EVs. The degradation cost of EVs and ESS $C_{t}^{\text {EVIESS }}$ is also considered in the energy management scheme, which is given by

$$
C_{t}^{\mathrm{EV} / \mathrm{ESS}}=\frac{C^{\mathrm{bat}}}{L^{\mathrm{c}} E d^{\mathrm{DoD}}}\left(P_{t}^{\mathrm{ch}} \eta^{\mathrm{ch}}+\frac{P_{t}^{\mathrm{dsh}}}{\eta^{\mathrm{dhh}}}\right)
$$

where $C^{\text {bat }}$ is the total investment on the battery; $L^{\mathrm{c}}$ is the charge/discharge cycle number during the life cycle of the battery; $E$ denotes the battery capacity; and $d^{\mathrm{DoD}}$ is the depth of discharge (DoD). $P_{t}^{\mathrm{ch}}, P_{t}^{\mathrm{dsh}}$ and $\eta^{\mathrm{ch}}, \eta^{\mathrm{dsh}}$ represent the charge/discharge power and efficiency of the EV/ESS, respectively.

The power limit constraints of EV/ESS $i$ are given as follows:

$$
\begin{aligned}
& 0 \leq P_{i, t}^{\mathrm{ch}} \leq P_{\mathrm{max}}^{\mathrm{ch},} \\
& 0 \leq P_{i, t}^{\mathrm{dsh}} \leq P_{\max }^{\mathrm{dsh}}
\end{aligned}
$$

The definition of the state of charge (SoC) of EV/ESS $i$ represents the energy characteristics as follows:

$$
\mathrm{SoC}_{i, t}^{\mathrm{EV} / E S S}=\mathrm{SoC}_{i, t-1}^{\mathrm{EV} / E S S}+\frac{1}{E_{i}}\left(P_{i, t}^{\mathrm{ch}} \eta^{\mathrm{ch}}-\frac{P_{i, t}^{\mathrm{dsh}}}{\eta^{\mathrm{dsh}}}\right) \Delta t
$$

The energy limit constraints include the SoC limit and the final $\mathrm{SoC}$ requirement.

$$
\mathrm{SoC}_{\mathrm{EV} / \mathrm{ESS}}^{\min } \leq \mathrm{SoC}_{i, t}^{\mathrm{EV} / \mathrm{ESS}} \leq \mathrm{SoC}_{\mathrm{EV} / \mathrm{ESS}}^{\max }
$$

In addition, the SoC requirement of EV $i$ must be met before the occupant leaves, as shown by the following constraint:

$$
\mathrm{SoC}_{i, t_{a}}^{\mathrm{EV}}+\sum_{t=t_{a}}^{t_{t}} \frac{1}{E_{i}}\left(P_{i, t}^{\mathrm{EVch}} \eta^{\mathrm{EVch}}-\frac{P_{i, t}^{\mathrm{EVdsh}}}{\eta^{\mathrm{EVdsh}}}\right) \Delta t \geq \mathrm{SoC}_{i}^{\mathrm{EV}}
$$

where $t_{a}$ and $t_{l}$ are the on-grid time and off-grid time, respectively. $\mathrm{SoC}_{i, t_{a}}^{\mathrm{EV}}$ is the initial $\mathrm{SoC}$ at the on-grid time, and $\mathrm{SoC}_{i}^{\mathrm{EVe}}$ is the expected value for the EV occupant at the off-grid time.

$$
\mathrm{SoC}_{i, 0}^{\mathrm{ESS}}=\mathrm{SoC}_{i, T}^{\mathrm{ESS}}
$$

Constraint (11f) on an ESS ensures that the total energy available in the battery at the end of the time horizon is the same as it was at the beginning.

\section{Uncertainty Modeling of PVs and Loads}

Considering the uncertainty of PVs and loads, the forecast values take into account the forecast error based on the normal distribution. The actual power of PVs and loads are expressed as:

$$
\begin{aligned}
P_{b, t, s}^{\mathrm{PV}} & =P_{b, t}^{\mathrm{PV}}+P_{b, t, s}^{\mathrm{PVerr}} \\
P_{b, t, s}^{\mathrm{load}} & =P_{b, t}^{\mathrm{load}}+P_{b, t, s}^{\mathrm{loader}}
\end{aligned}
$$

where $P_{b, t}^{\mathrm{PV}}, P_{b, t}^{\mathrm{load}}$ are the predicted values of PVs and loads in 
MECM $b$, respectively, and $P_{b, t, s}^{\mathrm{PVerr}}$ and $P_{b, t, s}^{\text {loader }}$ are the forecast errors of PVs and loads in scenario $s$.

In this paper, representative scenarios are generated based on Monte Carlo sampling and K-mean cluster technique [29].

\section{MECMS OPTIMAL SCHEDULING MODEL}

\section{A. Multi-energy sharing model}

\section{1) Objective function}

In consideration of the uncertainties in the load and solar power, scenario-based stochastic programming is adopted. On the basis of the multi-energy sharing strategy, the interconnected energy system of multiple MECMs aims to minimize the total expected operation cost in $N s$ representative scenarios, including the cost of electricity trading with the utility grid, power generation cost, network cost and degradation cost of EVs and ESS. The objective function is expressed as follows:

$$
\begin{gathered}
\min C=\sum_{s=1}^{N s} \sum_{b=1}^{N b} \sum_{t=1}^{N T} \pi_{s}\left(C_{b, t, s}^{\mathrm{grid}}+C_{b, t, s}^{\mathrm{cchp}}+\gamma_{b, t, s}+C_{b, t, s}^{\mathrm{EV}}+C_{b, t, s}^{\mathrm{ESS}}\right) \\
=\sum_{s=1}^{N s} \sum_{b=1}^{N b} \sum_{t=1}^{N T} \pi_{s}\left(\left(c_{t}^{\mathrm{gb}} P_{b, t . s}^{\mathrm{gb}}-c_{t}^{\mathrm{gs}} P_{b, t, s}^{\mathrm{gs}}\right) \Delta t+c^{\mathrm{gas}} F_{b, t, s}^{\mathrm{gas}}\right. \\
+\alpha^{\mathrm{p}}\left(P_{b, t, s}^{\mathrm{ex}}\right)^{2} \Delta t+\alpha^{\mathrm{h}}\left(H_{b, t, s}^{\mathrm{ex}}\right)^{2} \Delta t \\
\left.+C_{b, t, s}^{\mathrm{EV}}+C_{b, t, s}^{\mathrm{ESS}}\right)
\end{gathered}
$$

where $\pi_{s}$ is the probability of scenario $s$; Ns is the set of respective scenarios; $N b$ is the set of MECMs; $c_{t}^{\mathrm{gb}}, c_{t}^{\mathrm{gs}}$ and $c^{\text {gas }}$ are the buying, selling prices of the utility grid and the price of natural gas, respectively; $P_{b, t . s}^{\mathrm{gb}}$ is the electricity purchased from the utility grid; and $P_{b, t . s}^{\mathrm{gs}}$ is the electricity fed back to the utility grid. The network cost of the distribution grid and heat pipes is denoted by $\gamma_{b, t, s}[30] ; \alpha^{\mathrm{p}}, \alpha^{\mathrm{h}}$ are the corresponding cost coefficients.

\section{2) Power balance}

The electrical power balance constraint for each MECM $b$ in scenario $s$ is denoted as

$$
\begin{aligned}
& \left(P_{b, t, s}^{\mathrm{short}}-P_{b, t, s}^{\mathrm{sur}}\right)+P_{b, t, s}^{\mathrm{PV}}+P_{b, t, s}^{\mathrm{mt}}=P_{b, t, s}^{\mathrm{hp}}+P_{b, t, s}^{\mathrm{load}} \\
& +\sum_{i=1}^{N b_{v}}\left(P_{i, t, s}^{\mathrm{EVch}}-P_{i, t, s}^{\mathrm{EVdsh}}\right)+\left(P_{b, t, s}^{\mathrm{ESSch}}-P_{b, t, s}^{\mathrm{ESSdsh}}\right)
\end{aligned}
$$

In addition to electrical energy sharing among MECMs, the remaining surplus/shortage of electrical power can be traded with the utility grid, which is described as follows:

$$
\begin{gathered}
P_{b, t, s}^{\mathrm{short}}=P_{b, t, s}^{\mathrm{mb}}+P_{b, t, s}^{\mathrm{gb}} \\
P_{b, t, s}^{\mathrm{sur}}=P_{b, t, s}^{\mathrm{ms}}+P_{b, t, s}^{\mathrm{gs}}
\end{gathered}
$$

The total shortage of electrical power is compensated by other MECMs and the utility grid; $P_{b, t, s}^{\mathrm{mb}}$ is the electricity purchased from other MECMs; and $P_{b, t, s}^{\mathrm{ms}}$ is the electricity sold to other MECMs.

Assuming that there is no transmission loss in the electrical network yields

$$
P_{b, t, s}^{\mathrm{ex}}=P_{b, t, s}^{\mathrm{mb}}-P_{b, t, s}^{\mathrm{ms}}
$$

where $P_{b, t, s}^{\mathrm{ex}}$ denotes the electrical power shared with others.

The heat balance can be achieved by trading heat with other MECMs, as indicated by

$$
H_{b, t, s}^{\mathrm{mb}}-H_{b, t, s}^{\mathrm{ms}}+H_{b, t, s}^{\mathrm{mt}}=H_{b, t, s}^{\mathrm{ac}}+H_{b, t, s}^{\mathrm{hx}}
$$

We regard thermal networks as heat storage, and the actual thermal power shared with other MECMs considering the heat loss in heat pipes $H_{b, t, s}^{\mathrm{ex}}$ is defined as

$$
H_{b, t, s}^{\mathrm{ex}}=(1+\beta) H_{b, t, s}^{\mathrm{mb}}-(1-\beta) H_{b, t, s}^{\mathrm{ms}}
$$

where $\beta$ is the heat loss coefficient.

Finally, the electrical and thermal power balances of energy sharing among $\mathrm{Nb} \mathrm{MECMs}$ are given by

$$
\begin{aligned}
& \sum_{b=1}^{N_{b}} P_{b, t, s}^{\mathrm{ex}}=0 \\
& \sum_{b=1}^{N_{b}} H_{b, t, s}^{\mathrm{ex}}=0
\end{aligned}
$$

\section{3) Power limit}

We define binary variables $x_{b, t, s}^{P}$ and $x_{b, t, s}^{h}$ that represent the directions of transmission of shared electrical power and thermal power, respectively. If $x_{b, t, s}^{P}=1$, the MECM imports electrical power to fulfill the energy demand shortage; otherwise, $x_{b, t, s}^{P}=0$. The definition for heat flow is the same.

The constraints for the capacity of an electrical line are presented as follows:

$$
\begin{gathered}
0 \leq P_{b, t, s}^{\mathrm{mb}} \leq x_{b, t, s}^{P} P_{\max }^{\mathrm{line}} \\
0 \leq P_{b, t, s}^{\mathrm{ms}} \leq\left(1-x_{b, t, s}^{P}\right) P_{\max }^{\mathrm{line}}
\end{gathered}
$$

We assume that the amount of electricity in an MECM that can interact with the utility grid has no limit, and the Big M method is proposed to ensure the direction of power flow. This condition is consistent with that of an MECM interacting with other MECMs.

$$
\begin{gathered}
0 \leq P_{b, t, s}^{\mathrm{gb}} \leq x_{b, t, s}^{P G} M \\
0 \leq P_{b, t, s}^{\mathrm{gs}} \leq\left(1-x_{b, t, s}^{P G}\right) M
\end{gathered}
$$

Similarly, the limitation of heat pipe capacity is described as

$$
\begin{gathered}
0 \leq H_{b, t, s}^{\mathrm{mb}} \leq x_{b, t, s}^{h} H_{\mathrm{max}}^{\mathrm{line}} \\
0 \leq H_{b, t, s}^{\mathrm{ms}} \leq\left(1-x_{b, t, s}^{h}\right) H_{\max }^{\mathrm{line}}
\end{gathered}
$$

\section{B. Distributed Optimization of the Energy Sharing Problem}

\section{1) Problem Decoupling through the ADMM}

The multi-energy sharing model aims to minimize the total operating cost, and the objective function (13) can be expressed as the sum of the operating costs of each MECM.

$$
\begin{aligned}
& \min C=\sum_{s=1}^{N s} \sum_{b=1}^{N b} \pi_{s} C_{b, s}\left(\mathbf{x}_{b, s}\right) \\
& =\sum_{s=1}^{N s} \sum_{b=1}^{N b} \pi_{s}\left(\sum_{t=1}^{N T} C_{b, t, s}^{\text {grid }}+C_{b, t, s}^{\mathrm{cchp}}+\gamma_{b, t, s}+C_{b, t, s}^{\mathrm{EV}}+C_{b, t, s}^{\mathrm{ESS}}\right) \\
& \text { s.t. } \quad(1)-(9),(11),(14)-(19)
\end{aligned}
$$

We take one column of the matrix representing the multi 
energy expected to be shared by MECM $b$ :

$$
\mathbf{x}_{b, s}=\left[\boldsymbol{P}_{b, s}^{\mathrm{gb}}, \boldsymbol{P}_{b, s}^{\mathrm{gs}}, \boldsymbol{P}_{b, s}^{\mathrm{mt}}, \boldsymbol{P}_{b, s}^{\mathrm{hp}}, \boldsymbol{P}_{b, s}^{\mathrm{EV}}, \boldsymbol{P}_{b, s}^{\mathrm{ESS}}, \boldsymbol{P}_{b, s}^{\mathrm{ex}}, \boldsymbol{H}_{b, s}^{\mathrm{ex}}\right]^{T}
$$

where $\boldsymbol{P}_{b, s}^{\mathrm{gb}}=\left[P_{b, 1, s}^{\mathrm{gb}}, \ldots, P_{b, T, s}^{\mathrm{gb}}\right]$ denotes the electricity purchased from the grid during the scheduling period in scenario $s$ and the representation of other variables is the same.

Note that coupling constraints (17a) and (17b) guarantee the supply-demand balance of multi-energy sharing, and then we define the variable matrix to represent the expected shared electrical and thermal energy of multiple MECMs during the scheduling period.

$$
\mathbf{x}_{s}^{\mathrm{ex}}=\left[\begin{array}{ccccc}
P_{1,1, s}^{\mathrm{ex}} & \cdots & P_{b, 1, s}^{\mathrm{ex}} & \cdots & P_{N b, 1, s}^{\mathrm{ex}} \\
\vdots & \ddots & \vdots & \ddots & \vdots \\
P_{1, N T, s}^{\mathrm{ex}} & \cdots & P_{b, T, s}^{\mathrm{ex}} & \cdots & P_{N b, N T, s}^{\mathrm{ex}} \\
H_{1,1, s}^{\mathrm{ex}} & \cdots & H_{b, 1, s}^{\mathrm{ex}} & \cdots & H_{N b, 1, s}^{\mathrm{ex}} \\
\vdots & \ddots & \vdots & \ddots & \vdots \\
H_{1, N T, s}^{\mathrm{ex}} & \cdots & H_{b, N T, s}^{\mathrm{ex}} & \cdots & H_{N b, N T, s}^{\mathrm{ex}}
\end{array}\right]
$$

We take one column of the matrix representing the expected multi-energy sharing by MECM $b$.

$$
\mathbf{x}_{b, s}^{\mathrm{ex}}=\left[P_{b, 1, s}^{\mathrm{ex}}, P_{b, 2, s}^{\mathrm{ex}}, \ldots, P_{b, N T, s}^{\mathrm{ex}}, H_{b, 1, s}^{\mathrm{ex}}, H_{b, 2, s}^{\mathrm{ex}}, \ldots, H_{b, N T, s}^{\mathrm{ex}}\right]^{T}(21 \mathrm{c})
$$

The auxiliary variable matrix $\mathbf{z}_{s}=\left[z_{1, s}, \cdots, z_{2 N T, s}\right]^{T}$ has the dimensions $2 N T \times N b$, and $\mathbf{z}_{b, s}$ is the column vector corresponding to MECM $b$ in scenario $s$. The value function $g\left(\mathbf{z}_{\mathrm{s}}\right)$ in scenario $s$ is introduced to construct the general form of ADMM; then, we obtain

$$
\begin{aligned}
& g\left(\mathbf{z}_{s}\right)=\left\{\begin{array}{cc}
0 & \mathbf{z}_{s} \in C_{s} \\
+\infty & \mathbf{z}_{s} \notin C_{s}
\end{array}\right. \\
& C_{s}=\left\{\mathbf{z}_{s} \mid \sum_{b=1}^{N b} z_{i, b, s}=\mathbf{0}\right\} \quad i \in\{1, \ldots, 2 T\}
\end{aligned}
$$

Subsequently, (20) can be written in ADMM form [14] as

$$
\begin{aligned}
& \min \sum_{s=1}^{N s} \pi_{s} \sum_{b=1}^{N b} C_{b, s}\left(\mathbf{x}_{b, s}\right)+g\left(\mathbf{z}_{s}\right) \\
& \text { s.t. } \quad \mathbf{x}_{b, s}^{\mathrm{ex}}=\mathbf{z}_{b, s} \\
& \quad \mathbf{x}_{b, s} \in \mathbb{Q}_{b, s}
\end{aligned}
$$

The optimization problem (23) can be decoupled into $\mathrm{Nb}$ distributed subproblems iteratively solved by each MECM, where $\mathbb{Q}_{b, s}$ is the constraint set for MECM $b$ in scenario $s$. The problem can be formulated as

$$
\begin{aligned}
& \mathbf{x}_{b}^{(k+1)}= \underset{\mathbf{x}_{b}}{\arg \min } \sum_{s=1}^{N s} \pi_{s}\left(C_{b, s}\left(\mathbf{x}_{b, s}\right)+\mathbf{y}_{b, s}^{(k) T}\left(\mathbf{x}_{b, s}^{\mathrm{ex}}-\mathbf{z}_{b, s}^{(k)}\right)\right. \\
&\left.+(\rho / 2)\left\|\mathbf{x}_{b, s}^{\mathrm{ex}}-\mathbf{z}_{b, s}^{(k)}\right\|_{2}^{2}\right) \\
& \mathbf{z}^{(k+1)}=\underset{\mathbf{z}}{\arg \min } \sum_{s=1}^{N s} \pi_{s}\left(g\left(\mathbf{z}_{s}\right)+\sum_{b=1}^{N b} \mathbf{y}_{b, s}^{(k) T}\left(\mathbf{x}_{b, s}^{\mathrm{ex}(k)}-\mathbf{z}_{b, s}\right)\right. \\
& \\
&\left.+(\rho / 2) \sum_{b=1}^{N b}\left\|\mathbf{x}_{b, s}^{\mathrm{ex}(k)}-\mathbf{z}_{b, s}\right\|_{2}^{2}\right) \\
& \mathbf{y}_{b, s}^{(k+1)}= \mathbf{y}_{b, s}^{(k)}+\rho\left(\mathbf{x}_{b, s}^{\mathrm{e}(k+1)}-\mathbf{z}_{b, s}^{(k+1)}\right)
\end{aligned}
$$

Lagrange multipliers $\boldsymbol{\mu}_{s}=\left[\mu_{1, s}, \cdots, \mu_{2 N T, s}\right]$ are used to relax the equality constraint, and the Lagrangian problem of (25a) is described as

$$
\begin{aligned}
L(\mathbf{z}, \boldsymbol{\mu}) & =\sum_{s=1}^{N s} \pi_{s}\left(\tilde{g}\left(\overline{\mathbf{z}}_{s}\right)+\sum_{b=1}^{N b} \mathbf{y}_{b, s}^{(k) T}\left(\mathbf{x}_{b, s}^{\mathrm{ex}(k)}-\mathbf{z}_{b, s}\right)\right. \\
& \left.+(\rho / 2) \sum_{b=1}^{N b}\left\|\mathbf{x}_{b, s}^{\operatorname{ex}(k)}-\mathbf{z}_{b, s}\right\|_{2}^{2}+\boldsymbol{\mu}_{s}\left(\overline{\mathbf{z}}_{s}-\mathbf{z}_{s}^{\prime}\right)\right)
\end{aligned}
$$

where $\mathbf{z}_{s}^{\prime}=\frac{1}{N b}\left[\sum_{b=1}^{N b} z_{b, 1, s}, \cdots, \sum_{b=1}^{N b} z_{b, 2 N T, s}\right]$ and $\mathbf{z}, \boldsymbol{\mu}$ represent the set of profiles $\mathbf{z}_{s}, \boldsymbol{\mu}_{s}$ in each scenario.

Note that the fixed $\overline{\mathbf{z}}_{s}$ is scalar; thus, the necessary and 
sufficient condition for optimality of the convex problem is $\nabla_{\mathbf{z}} L(\mathbf{z}, \boldsymbol{\mu})=0$, which is given in detail as:

$$
\nabla_{\mathbf{z}} L(\mathbf{z}, \boldsymbol{\mu})=\pi_{s}\left(\mathbf{y}_{b, s}^{(k)}+\rho\left(\mathbf{x}_{b, s}^{\mathrm{ex}(k)}-\mathbf{z}_{b, s}\right)+\frac{\boldsymbol{\mu}_{s}}{N b}\right)=0
$$

With the average (over $b=1, \ldots, N b)$ of $\mathbf{y}_{s}$ and $\mathbf{x}_{s}^{\mathrm{ex}}$, the averaging conditions in each scenario for optimality yield

$$
\mathbf{y}_{b, s}^{(k)}+\rho\left(\mathbf{x}_{b, s}^{\mathrm{ex}(k)}-\mathbf{z}_{b, s}\right)=\overline{\mathbf{y}}_{s}^{(k)}+\rho\left(\overline{\mathbf{x}}_{s}^{\mathrm{ex}}-\overline{\mathbf{z}}_{s}\right)
$$

As $\overline{\mathbf{z}}_{s} \equiv 0$ during the iteration, (25e) is employed in (24), and the ADMM algorithm for solving (20) can be simplified further to the exchange form in Chapter 7 in [14], which is described as

$$
\begin{gathered}
\mathbf{x}_{b}^{(k+1)}=\underset{\mathbf{x}_{b}}{\arg \min } \sum_{s=1}^{N s} \pi_{s}\left(C_{b, s}\left(\mathbf{x}_{b . s}\right)+\overline{\mathbf{y}}_{s}^{(k)} \mathbf{x}_{b, s}^{\mathrm{ex}}\right. \\
\left.\quad+(\rho / 2)\left\|\mathbf{x}_{b . s}^{\mathrm{ex}}-\left(\mathbf{x}_{b, s}^{\mathrm{ex}(k)}-\overline{\mathbf{x}}_{s}^{\mathrm{ex}(\mathrm{k})}\right)\right\|_{2}^{2}\right) \\
\text { s.t. } \quad \mathbf{x}_{b, s} \in \mathbb{Q}_{b, s} \\
\quad \overline{\mathbf{y}}_{s}^{(k+1)}=\overline{\mathbf{y}}_{s}^{(k)}+\rho \overline{\mathbf{x}}_{s}^{\mathrm{ex}(k+1)}
\end{gathered}
$$

The objective function is convex, but binary variables exist in constraints (18a)-(19b). However, the optimization problem (20) can be carried out exactly since the nonconvex constraints are Boolean [14].

In our case, the primal residual and dual residual in each scenario are applied as the convergence criterion of the ADMM algorithm, which guarantees the power balance of energy sharing:

$$
\begin{aligned}
\left\|\gamma^{(k)}\right\|_{2}^{2} & =\left\|\frac{1}{N b} \sum_{b=1}^{N b} x_{b, s}^{\mathrm{ex}(k)}\right\|_{2}^{2} \leq \varepsilon^{\mathrm{pri}} \\
\left\|v^{(k)}\right\|_{2}^{2} & =\left\|v_{1, s}^{(k)}, \cdots, v_{N b, s}^{(k)}\right\|_{2}^{2} \leq \varepsilon^{\text {dual }}
\end{aligned}
$$

where

$$
v_{b, s}^{(k)}=\rho \frac{1}{N_{b}}\left(\mathbf{x}_{b, s}^{\mathrm{ex}(k)}-\mathbf{x}_{b, s}^{\mathrm{ex}(k-1)}+\left(\overline{\mathbf{x}}_{s}^{\mathrm{ex}(k-1)}-\overline{\mathbf{x}}_{s}^{\mathrm{ex}(\mathrm{k})}\right)\right)
$$

With these simplifications, the distributed optimization is formulated by Algorithm 1 as follows.

\begin{tabular}{l}
\hline Algorithm 1 Distributed optimization \\
\hline Initialize $\mathbf{x}_{b, s}^{\mathrm{ex}(0)}$ and publish $\rho, \overline{\mathbf{y}}_{s}^{(0)}, \overline{\mathbf{x}}_{s}^{\mathrm{ex}(0)}$ to each \\
MECM in each scenario. \\
Repeat \\
$>$ Local optimization at each MECM \\
$\qquad \mathbf{x}_{b}^{(k+1)}=\underset{\mathbf{x}_{b}}{\arg \min } \sum_{s=1}^{N s} \pi_{s}\left(C_{b, s}\left(\mathbf{x}_{b, s}\right)+\overline{\mathbf{y}}_{s}^{(k)} \mathbf{x}_{b, s}^{\mathrm{ex}}\right.$ \\
$\left.\quad+(\rho / 2)\left\|\mathbf{x}_{b, s}^{\mathrm{ex}}-\left(\mathbf{x}_{b . s}^{\mathrm{ex}(k)}-\overline{\mathbf{x}}_{s}^{\mathrm{ex}(k)}\right)\right\|_{2}^{2}\right)$ \\
$\quad \quad \mathbf{x}_{b, s} \in \mathbb{Q}_{b, s}$ \\
submit $\mathbf{x}_{b, s}^{\mathrm{ex}(k+1)}$ to RIES operator \\
RIES operator coordination \\
After receiving $\mathbf{x}_{b, s}^{\mathrm{e}(k+1)}$ from each MECM, RIES \\
operator calculate the average over MECMs
\end{tabular}

$$
\overline{\mathbf{x}}_{s}^{\mathrm{ex}(\mathrm{k}+1)}=\frac{1}{N b} \sum_{b=1}^{N b} \mathbf{x}_{b, s}^{\mathrm{ex}(k)}
$$

Update and publish dual-price

$$
\overline{\mathbf{y}}_{s}^{(k+1)}=\overline{\mathbf{y}}_{s}^{(k)}+\rho \overline{\mathbf{x}}_{s}^{\mathrm{ex}(k)}
$$

until convergence condition is verified

$$
\begin{gathered}
\left\|\gamma^{(k)}\right\|_{2}^{2} \leq \varepsilon^{\text {pri }} \\
\left\|\delta^{(k)}\right\|_{2}^{2} \leq \varepsilon^{\text {dual }}
\end{gathered}
$$

\section{Economic interpretation}

Problem (20) is a sharing problem where the components of $\mathbf{x}_{b}^{\mathrm{ex}}$ represent the quantities of electrical and thermal power shared among $N b$ MECMs in each scenario, and the equilibrium constraints $\sum_{b=1}^{N_{b}} P_{b, t}^{\text {ex }}=0$ and $\sum_{b=1}^{N_{b}} H_{b, t}^{\text {ex }}=0$ can be interpreted as market clearing of shared energy.

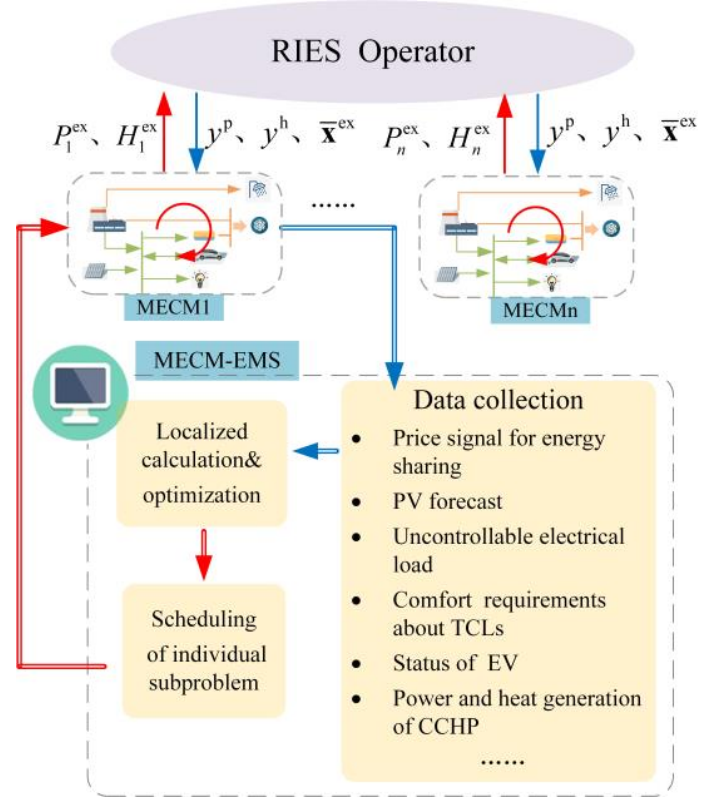

Fig. 2. Iterative data exchange between the local optimization of each individual MECM, and the centralized coordination from RIES Operator

As shown in Fig. 2, the ADMM algorithm can be interpreted as a combination of localized optimization and centralized coordination. At each iteration, each MECM-EMS solves the individual optimization subproblem (26) based on the detailed information of end users and day-ahead price locally and submits the expected shared electrical and thermal power vector $\mathbf{x}_{b}^{\mathrm{ex}(k)}$.The cost $\overline{\mathbf{y}}^{k} \mathbf{x}_{b}^{\mathrm{ex}}$ and the added penalty $(\rho / 2)\left\|\mathbf{x}_{b}^{\mathrm{ex}}-\left(\mathbf{x}_{b}^{\mathrm{ex}(k)}-\overline{\mathbf{x}}^{\mathrm{ex}(\mathrm{k})}\right)\right\|_{2}^{2}$ incentivize the MECM to shift its consumption from the pattern with the intention of minimizing $C_{b}\left(\mathbf{x}_{b}\right)$ toward the global optimum and committing to clear the market. The RIES operator computes the average shared energy $\overline{\mathbf{x}}^{\mathrm{ex}}$ and, more importantly, adjusts the price signal $\overline{\mathbf{y}}^{k}$ toward energy equilibrium depending on the average electrical or thermal power residual over all MECMs. This information is broadcast back to the MECM-EMSs handling the $x$-updates. 
The iteration stops when the clearing process is completed, and $\overline{\mathbf{y}}^{k}$ converges to the clearing price of electrical and thermal power for energy sharing.

\section{CASe Studies}

\section{A. Case parameters}

In our work, an RIES consisting of 6 interconnected MECMs is presented. Of these, 5 MECMs are grid-connected, and MECM 4 participates in electricity sharing but works in a grid-disconnected operation mode. The operation modes of each MECM are listed in Table II in detail. A CCHP system is present in each MECM, and the parameters of the CCHP systems are listed in Table III in detail. All MECMs are equipped with a heat pump with a capacity of $200 \mathrm{~kW}$, and $\mathrm{CoP}^{\text {hp }}$ is 2.6. The MECMs have different configurations with corresponding details, as shown in Table II. MECM 1 manages 200 EVs; PV panels are installed in MECM 2 and MECM4; and MECM4 is equipped with an ESS. Each EV has a capacity of $64 \mathrm{kWh}$ and is operated between $10 \%$ and $95 \%$ of the state of charge. The maximum charging and discharging power for an $\mathrm{EV}$ is $7 \mathrm{~kW}$ with an efficiency of 0.95 . The available time of EVs is sampled on the basis of Monte Carlo simulation according to [27]. We assume that the initial state of charge (SOC) at the on-grid time is 0.45 and that the expected SOC at the off-grid time is 0.9. The related parameters of the degradation cost of the ESS and EVs are from [2] and [28]. The PV power and fixed load data are presented in Section V.B, which are obtained from a typical summer day in China [26]. Normal distributions are used to simulate the PV $N\left(0,\left(0.2 P_{\mathrm{f}}^{\mathrm{pv}}\right)^{2}\right)$ and load $N\left(0,\left(0.1 P_{\mathrm{f}}^{\text {lad }}\right)^{2}\right)$ forecast error.

Ten representative scenarios are generated by K-mean method, and the probabilities of the scenarios are listed in Table IV. Assuming that a water heater with a capacity of $50 \mathrm{~L}$ is consistently full, its thermal capacitance and thermal resistance are $2.1 \times 10^{5} \mathrm{~J} /{ }^{\circ} \mathrm{C}$ and $3170^{\circ} \mathrm{C} / \mathrm{kW}$, respectively. The related HVAC parameters are obtained from [26]. $L^{\text {hvng }}$ is set as 9.97 $\mathrm{kWh} / \mathrm{m}^{3}$, and $c^{\mathrm{gas}}$ is set as $2.2 \mathrm{CNY} / \mathrm{m}^{3} . \alpha^{\mathrm{p}}$ and $\alpha^{\mathrm{h}}$ are set as $1.2 \times 10^{-4} \mathrm{CNY} /\left((\mathrm{kW})^{2} \mathrm{~h}\right)$. The day-ahead electricity price is illustrated in Fig. 10. The maximum electrical line capacity is $2000 \mathrm{~kW}$, and the maximum heat pipe capacity is $200 \mathrm{~kW}$.

TABLE II

Profiles of MECMs

\begin{tabular}{ccccccc}
\hline MECM & 1 & 2 & 3 & 4 & 5 & 6 \\
\hline \multicolumn{7}{c}{ Water heater } \\
\hline Quantity & 100 & 150 & 100 & 150 & 100 & 100 \\
$\theta^{\text {wh }}\left({ }^{\circ} \mathrm{C}\right)$ & {$[45,50]$} & {$[45,50]$} & {$[45,50]$} & {$[45,50]$} & {$[45,50]$} & 48 \\
\hline \multicolumn{7}{c}{ HVAC } \\
\hline Quantity & 120 & 100 & 80 & 100 & 80 & 80 \\
$\theta^{a}\left({ }^{\circ} \mathrm{C}\right)$ & {$[24,28]$} & {$[24,28]$} & {$[24,28]$} & {$[24,28]$} & {$[24,28]$} & 26 \\
\hline \multicolumn{7}{c}{$\mathbf{E V}$} \\
\hline Quantity & 200 & - & & & \\
\hline \multicolumn{7}{c}{ PV } \\
\hline Install & No & Yes & No & Yes & No & No \\
\hline \multicolumn{7}{c}{}
\end{tabular}

\begin{tabular}{lcccccc}
\hline \multicolumn{7}{c}{ ESS } \\
\hline Capacity/kWh & - & - & - & 5000 & - & - \\
$P_{\max }^{\text {ESSh/ESSdsh }} / \mathrm{kW}$ & - & - & - & 800 & - & - \\
$\eta^{\mathrm{ESSch/ESSdsh}}$ & - & - & - & 0.9 & - & - \\
\hline
\end{tabular}

\begin{tabular}{ccccccc}
\multicolumn{7}{c}{ Operation mode } \\
\hline Electricity & Yes & Yes & Yes & Yes & Yes & Yes \\
sharing & Yes & Yes & Yes & Yes & No & Yes \\
Heat sharing & Yes & Yes & Yes & No & Yes & Yes \\
Grid-connected & Yes
\end{tabular}

TABLE III

Related Parameters of CCHP system

\begin{tabular}{ccccccc}
\hline CCHP & 1 & 2 & 3 & 4 & 5 & 6 \\
\hline$P_{\max }^{\mathrm{mt}} / \mathrm{kW}$ & 200 & 200 & 300 & 200 & 300 & 300 \\
$H_{\max }^{\mathrm{mt}} / \mathrm{kW}$ & 300 & 300 & 400 & 300 & 400 & 400 \\
$\eta^{\mathrm{mte}}$ & 0.32 & 0.32 & 0.35 & 0.32 & 0.35 & 0.35 \\
$\eta^{\text {loss }}$ & 0.15 & 0.15 & 0.08 & 0.15 & 0.08 & 0.08 \\
$C_{\text {oph }}$ & 0.8 & 0.8 & 0.8 & 0.8 & 0.8 & 0.8 \\
$H_{\max }^{\mathrm{hx}} / \mathrm{kW}$ & 300 & 300 & 300 & 300 & 300 & 300 \\
$H_{\max }^{\mathrm{ac}} / \mathrm{kW}$ & 200 & 200 & 200 & 200 & 200 & 200 \\
$\mathrm{CoP}^{\mathrm{ac}}$ & 1.5 & 1.5 & 1.5 & 1.5 & 1.5 & 1.5 \\
\hline
\end{tabular}

TABLE IV

Probability of scenarios under the uncertainty of PV and load

\begin{tabular}{cccccc}
\hline Scenario & 1 & 2 & 3 & 4 & 5 \\
Probability & 0.2275 & 0.0825 & 0.0850 & 0.0975 & 0.1125 \\
\hline Scenario & 6 & 7 & 8 & 9 & 10 \\
Probability & 0.0625 & 0.0925 & 0.0825 & 0.0875 & 0.0700 \\
\hline
\end{tabular}

We set the penalty parameter $\rho$ as $10^{-3}$, and the convergence criterion $\varepsilon^{\text {pri }}$ and $\varepsilon^{\text {dual }}$ are set as $10^{-6}$.

\section{B. Case results}

The simulations are carried out in MATLAB platform using Gurobi [31] solver along with Yalmip. It runs on a computer featuring an Intel(R) Core(TM) i7-6700CPU @3.40GHz and 8 GB of RAM. The scheduling period is from 12:00 to 12:00 of the next day and a 30-min interval is used.

\section{1) MECM scheduling results}

The results presented here are the expected values for all scenarios. Fig. 3 presents the electric power balance of each MECM, where a positive value means power generation, while a negative value represents power consumption. Taking the MECM1 power distribution as an example, it is seen that on-grid EVs respond to the guidance of incentive price signals and operate in the discharged state. However, due to the SOC limitation of EVs, MECM1 only shares the power from EVs during 17:00-23:00, denoted by the sky blue bar (Pex). For the period 10:00-16:00, excess PV and CCHP power generated from MECM2 is shared among the MECMs. In addition, because MECM4 works in a grid-disconnected operation mode, we can see that the ESS chooses to discharge in a period of high price (10:00-14:00), and the surplus energy along with sufficient PV is shared to relieve load burden of utility grid is shared to relieve the load burden of the utility grid; in contrast, in the case of insufficient power from its own DERs, especially 
during the period of 23:00-7:00 when the ESS is charging, the energy demand needs to be met through energy sharing.
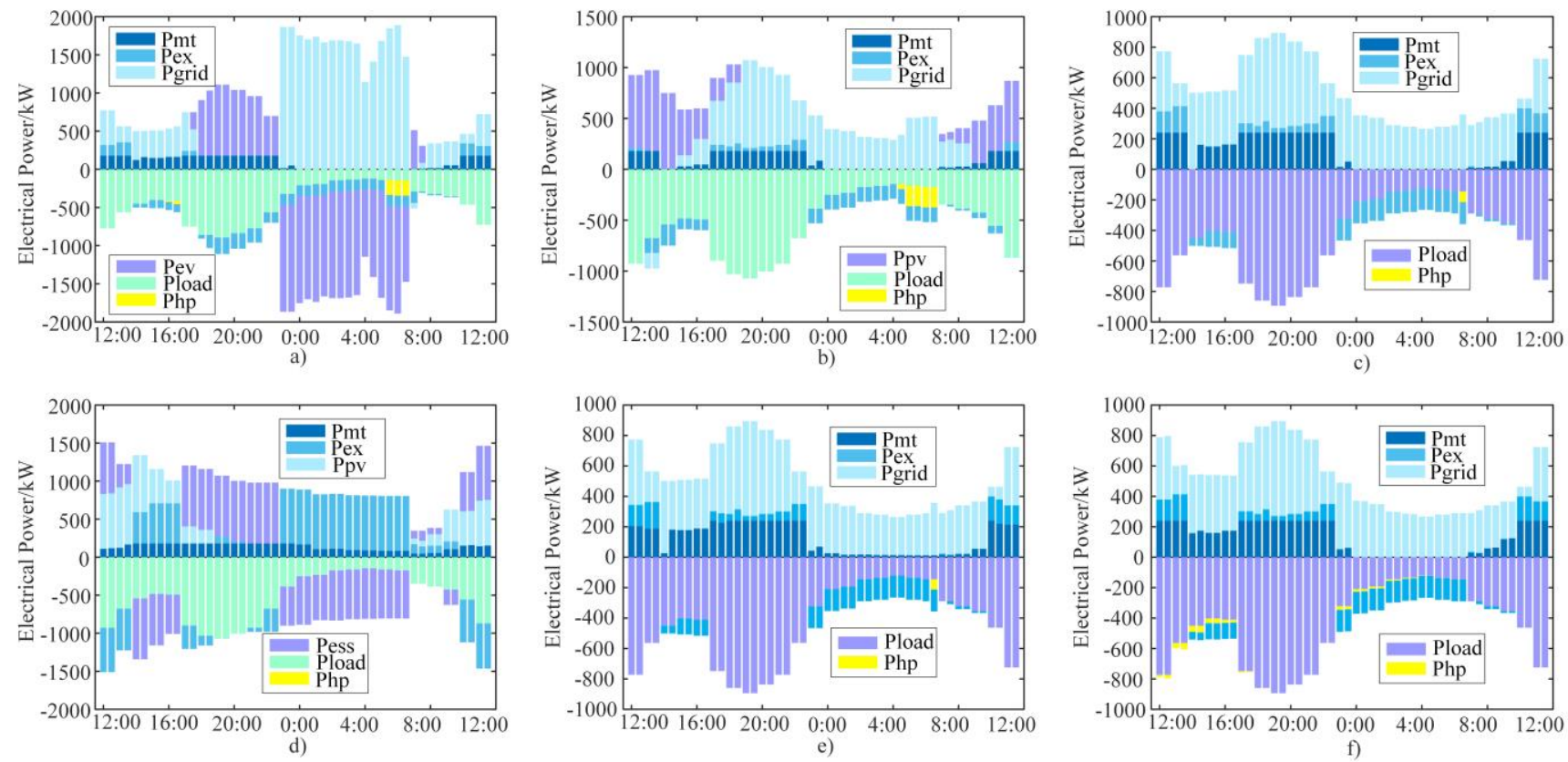

Fig. 3. Electrical power distribution situation of each MECM. a)-f) denote the power distribution of MECM 1-MECM 6, respectively.

MECM3 is taken as an example to further show the thermal power balance situation in Fig. 4. The dark blue bar (Hmt) represents the thermal power of the micro turbine, which has a high value during high electricity price periods (10:00-14:00 and 19:00-23:00). This verifies that the thermal power output is indirectly affected by the electricity price due to the electrical and thermal coupling of the device.

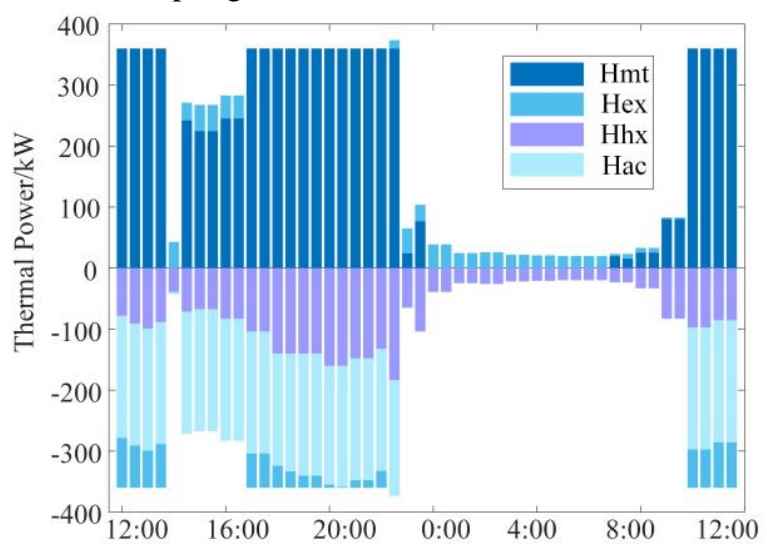

Fig. 4. Thermal power distribution situation of MECM3.

To achieve thermal energy balance, TCLs adapt to the energy supply by flexibly adjusting the heat demand. Fig. 5 illustrates the thermal consumption of the water heater and HVAC in the MECMs. The CCHP system provides heat for hot water, and cooling energy is supplied by the adsorption chillers of the CCHP system and heat pump using electricity. The heat pump, as a supplement to the cooling power of the CCHP system, consumes more power during low-price hours to precool within the comfort zone. Moreover, TCLs participate in IDR without violating physical and environmental requirements due to thermal inertia. We analyze the thermal energy sharing and IDR performance by comparing the thermal consumption of TCLs in MECM3 and MECM5. For the former, the MT can increase the thermal production and share surplus thermal energy to obtain the corresponding income, but for MECM5, which does not participate in thermal energy sharing, the high level of thermal power can only be absorbed by itself. Therefore, the hot water temperature is higher, while the difference in HVAC is not so large. MECM6 sets the TCLs' temperature to constant without using IDR. The thermal energy consumption is rigid and only related to user demand and ambient temperature.

2) Energy sharing scheduling results

The optimal scheduling results of shared electrical energy in scenario 1 are depicted in Fig. 6 and Fig. 7.

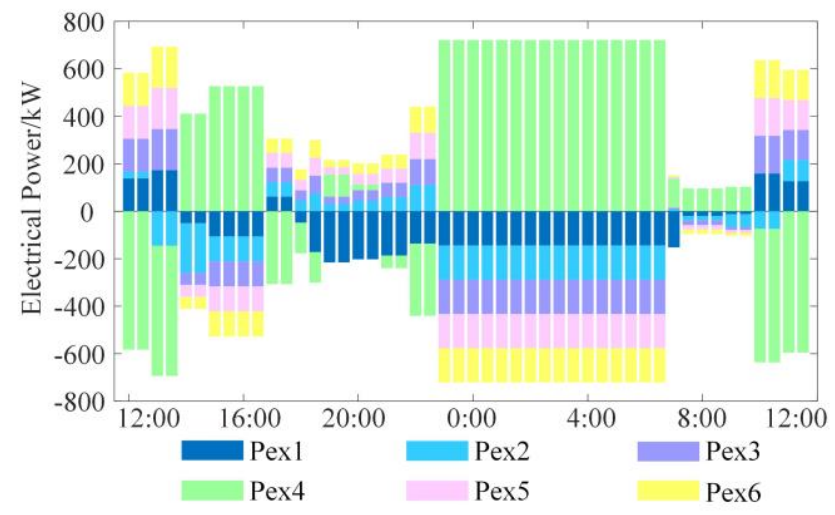

Fig. 6. Scheduling results of shared electrical energy.

In Fig. 6, the electricity from EVs and PV in MECM1 and MECM2 respectively can be shared among MECMs. MECM4 disconnects with utility, it sells surplus electricity in the process of energy sharing when PV and ESS is sufficient. However, limited by its DERs power level, MECM4 consumes shared 
energy to meet electricity demand during 15:00-17:00 and 23:00-7:00. According to the proposed framework, thermal

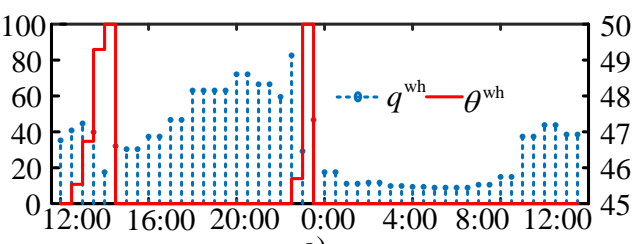

a)
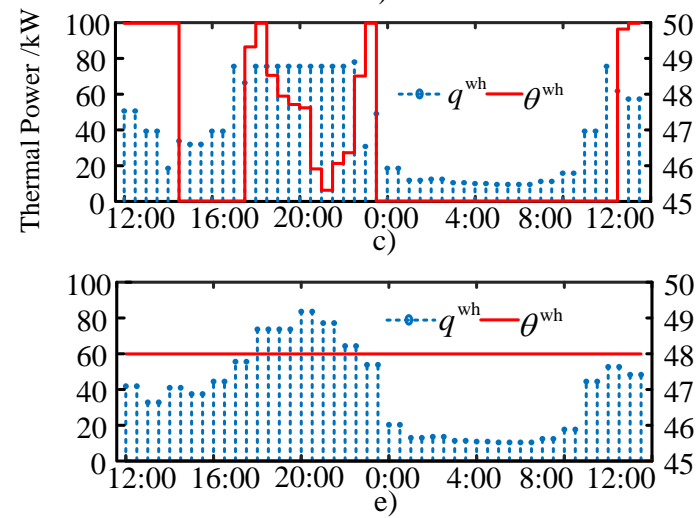

energy sharing of MECMs is coordinated within the RIES.
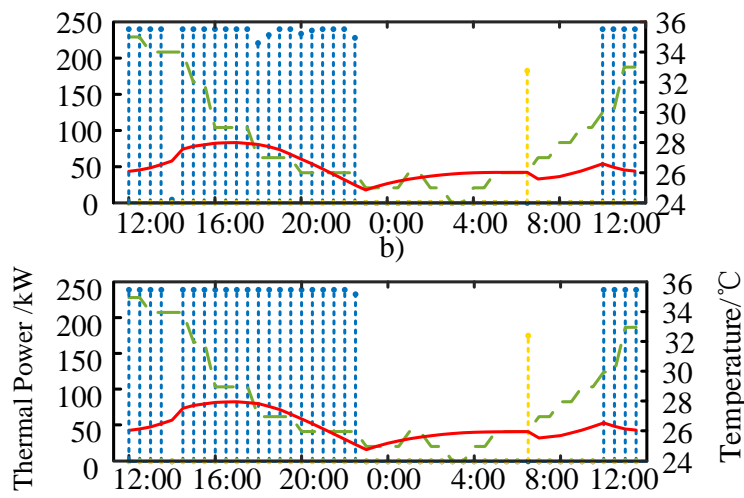

d)

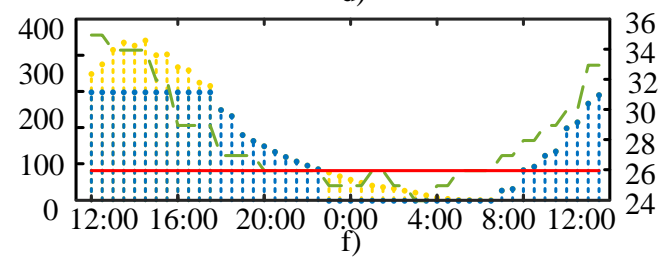

Fig. 5. Thermal power distribution situation of TCLs. a), c) e) show the thermal power situation of water heater in MECM3, MECM5, and MECM6 respectively; b), d) f) show the thermal power situation of HVAC in MECM3, MECM5, and MECM6 respectively.

From Fig. 7, we can find that the shortage thermal power of MECM1 and MECM2 are fulfilled by internal trading. Other MECMs can exchange their role depending on their thermal supply-demand relationship and responsibility for balancing thermal sharing. For example, MECM4 (the green bar) participates in thermal energy sharing as a seller during 10:00-14:00 and as a consumer during 15:00-17:00 and 23:00-9:00. It can be observed that the sum of shared electrical and thermal power are approximately zero, which confirms that the supply-demand balance constraints (17a) and (17b) are satisfied within the tolerance.

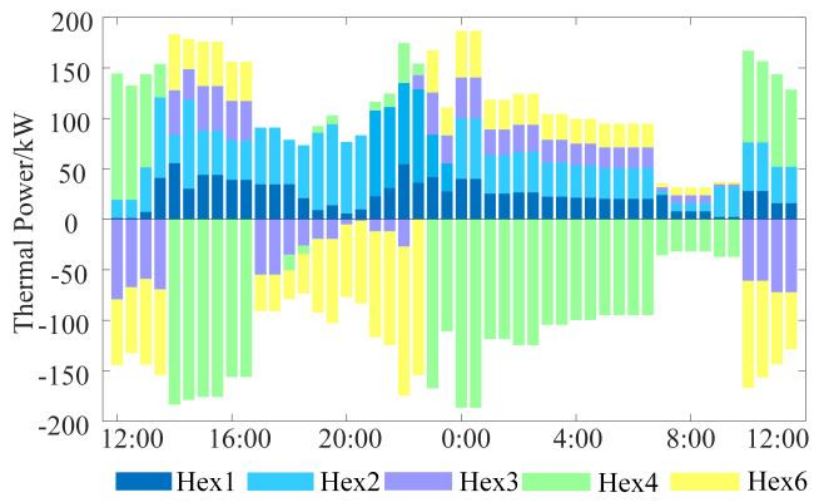

Fig. 7. Scheduling results of shared thermal energy.

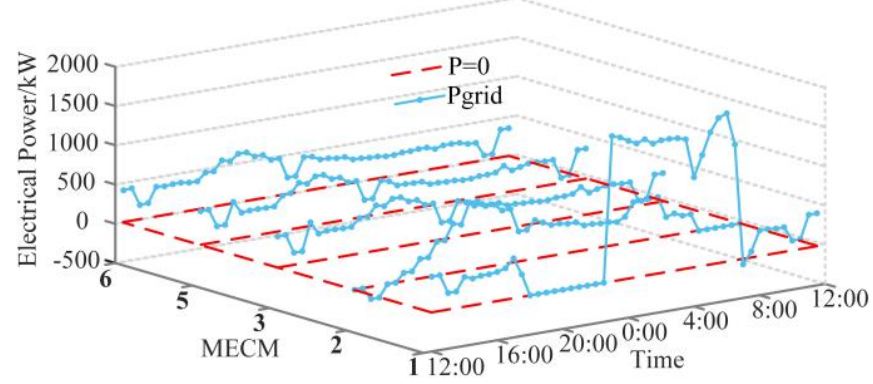

Fig. 8. Electrical power interaction between MECMs and utility grid.

Fig. 8 presents the power interaction between the MECMs and the utility grid in scenario 1 . Note that MECM2 and MECM1 sell power to the utility grid after performing internal energy sharing during 13:00-14:00 and 7:00-7:30, respectively. In contrast, the other MECMs still have power demand during these periods, and the surplus power is not fully shared internally but partly injected back to the utility grid because of the existence of network cost.
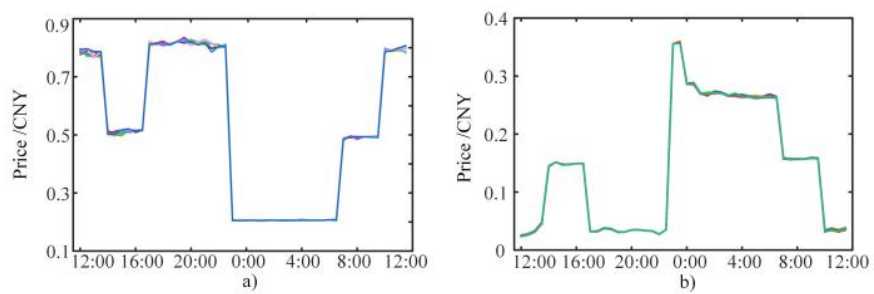

Fig. 9. Shared energy price in each scenario. a) shows the clearing price of electrical power for energy sharing; b) shows the clearing price of thermal power for energy sharing

In this paper, scenario-based stochastic programming is adopted to obtain the scheduling strategy. In terms of the clearing price for multi-energy sharing, the prices of shared electricity and heat in 10 scenario are illustrated in Fig. 9. 
Combined with the corresponding probabilities, the shared energy prices are expressed as:

$$
\overline{\mathbf{y}}=\frac{\sum_{b=1}^{N b}\left|\sum_{s=1}^{N s} \pi_{s} \overline{\mathbf{y}}_{s}^{T} \mathbf{x}_{b, s}^{\mathrm{ex}}\right|}{\sum_{b=1}^{N b}\left|\sum_{s=1}^{N s} \pi_{s} \mathbf{x}_{b, s}^{\mathrm{ex}}\right|}
$$

As mentioned in Section IV.C, the dual variable $\overline{\mathbf{y}}^{k}$ converges to the day-ahead clearing price for multi-energy sharing, as shown in Fig. 10. Observing the clearing prices of electrical and thermal power, the trend of shared electricity prices is similar to that of the market electricity price. The price trend of heat is the opposite because the CCHP system, as the device for coupling the electrical and thermal systems, will generate considerable electricity along with heat during high electricity price periods to reduce electricity purchasing costs. Thermal energy is shared at a low price and is regarded as a byproduct of electrical energy. Note that in the periods of 17:00-22:00 and 7:00-14:00, surplus power is traded internally to help reduce the total cost for purchasing power from the utility, which means that the sharing scheme is proactive and the shared electricity price is between $c_{t}^{\mathrm{gb}}$ and $c_{t}^{\mathrm{gs}}$. However, in terms of the periods of 23:00-7:00 and 14:00-17:00 when the total DER-generating power sources of the MECMs are insufficient, other MECMs can only purchase electricity from the utility and share with MECM4 to meet its demand, which causes a high network cost component in the marginal cost of the system, and the shared electricity price is higher than $c_{t}^{\mathrm{gb}}$.

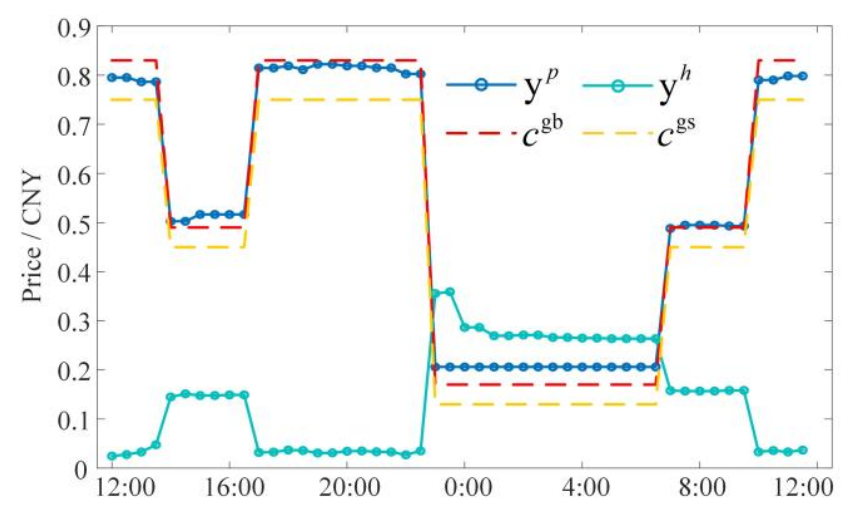

Fig. 10. Clearing price of electrical and thermal power for energy sharing.

In addition, our work performs the cost comparison of MECMs, as shown in Table V. In terms of the same configuration in the MECMs, the operation cost of MECM6 is much higher than that of MECM3, which confirms the effectiveness of IDR. In addition, with the assistance of IDR, participating in thermal energy sharing can also improve economic efficiency according to the cost of MECM5. MECM4 is disconnected from the utility grid and needs to be supplemented by energy sharing. Although network cost is unavoidable, the flexibility and adequacy of the RIES system are improved in this way. When an MECM is disconnected from the power grid, the energy supply can be supplemented through energy sharing among MECMs, thereby avoiding load shedding during DER shortage periods. Furthermore, we compare the cost of the case in which all MECMs work in an independent mode. Since MECM4 works in island mode and its own new energy resources cannot fully meet the power demand, the cost will not be discussed here. As shown in Table V, compared to the independent mode, the cost of each MECM in the multi-energy sharing mode is reduced. MECMs can choose more flexible means of operation, such as IDR and energy sharing. Additionally, shared electrical and thermal energy prices can guarantee the economic interests of individual MECMs.

TABLE V

Cost comparison of MECMs among MECMs and centralized optimization

\begin{tabular}{c|ccc}
\hline Cost(CNY) & Independent & $\begin{array}{c}\text { Multi-energy } \\
\text { sharing }\end{array}$ & Centralized \\
\hline MECM1 & 5850.7 & 5736.2 & - \\
MECM2 & 5464.0 & 5358.0 & - \\
MECM3 & 7303.5 & 7265.6 & - \\
MECM4 & - & 3594.8 & - \\
MECM5 & 7334.8 & 7289.1 & - \\
MECM6 & 7565.4 & 7432.3 & - \\
Total & - & 36676 & 36676 \\
\hline
\end{tabular}

3) Iterative process and ADMM performance

In this section, we discuss the iterative process and optimal scheduling results of energy sharing at 14:30 and 21:30 in scenario 1 as examples. In the iterative process of the ADMM algorithm, the buying price of the utility grid is selected as the initial value of the dual variable, and the initial heat price is taken as $0.4 \mathrm{CNY} / \mathrm{kWh}$. Convergence is achieved after 90 iterations.
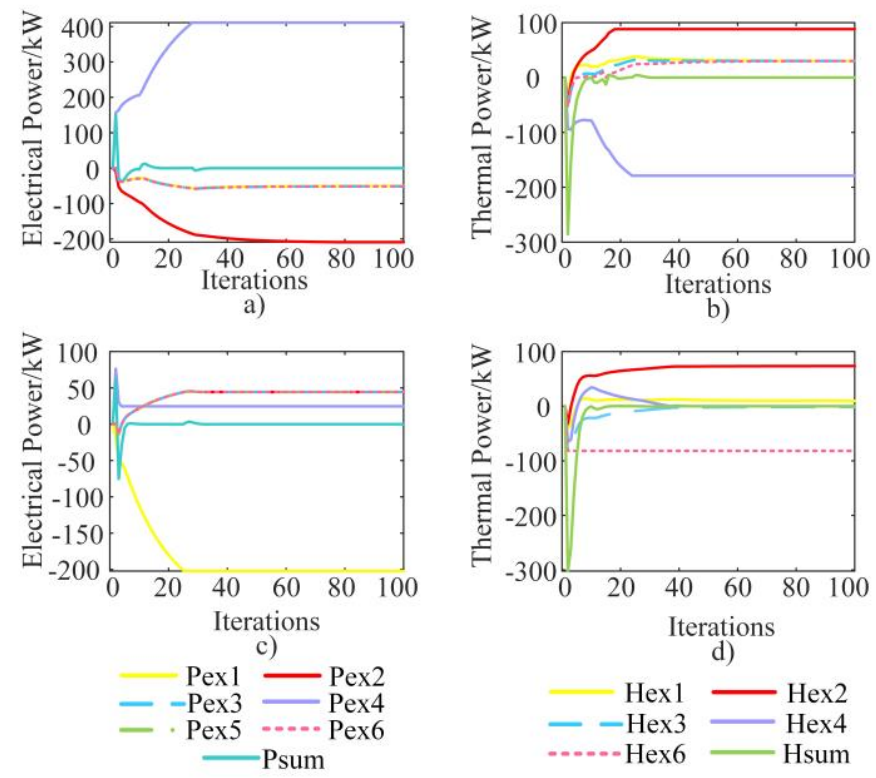

Fig. 11. Iterative process of shared energy at14:30 and 21:30. a), b) show the iterative process of shared electrical energy and thermal energy in each MECM at 14:30, respectively; c), d) show the iterative process of shared electrical energy and thermal energy in each MECM at 21:30, respectively.

The iterative processes of sharing electrical and thermal energy at 14:30 and 21:30 in each MECM are illustrated in Fig. 11. Table VI lists the convergence results of shared energy in detail. In terms of sharing electrical power at 14:30, the electricity purchased by MECM4 converges to $410.96 \mathrm{~kW}$, which is consistent with the electrical power sold by MECM1, 
3, 5, and 6 (all of them are $50.48 \mathrm{~kW}$ ) and MECM2 (209.04 $\mathrm{kW})$. MECM1, 2, 3 and 6 sell $30.29 \mathrm{~kW}, 88.19 \mathrm{~kW}, 30.23 \mathrm{~kW}$ and $30.15 \mathrm{~kW}$ thermal power to MECM4, respectively, and the total thermal power purchased by MECM2 is $178.86 \mathrm{~kW}$. Therefore, the balance of multi-energy sharing confirms that electrical and thermal energy is clear at 14:30 through the ADMM algorithm. Similarly, the shared electrical and thermal energy can achieve a balance at 21:30.

TABLE VI

Shared electrical and thermal power of each MECM at 14:30 and 21:30

\begin{tabular}{c|c|c|c|c}
\hline \multirow{2}{*}{ MECM } & \multicolumn{2}{|c|}{$14: 30$} & \multicolumn{2}{c}{$21: 30$} \\
\hline 1 & Pex & Hex & Pex & Hex \\
2 & -50.48 & 30.29 & -202.22 & 9.79 \\
3 & -209.04 & 88.19 & 44.40 & 73.46 \\
4 & -50.48 & 30.23 & 44.40 & -1.39 \\
5 & 410.96 & -178.86 & 24.62 & 0 \\
6 & -50.48 & - & 44.40 & - \\
\hline
\end{tabular}

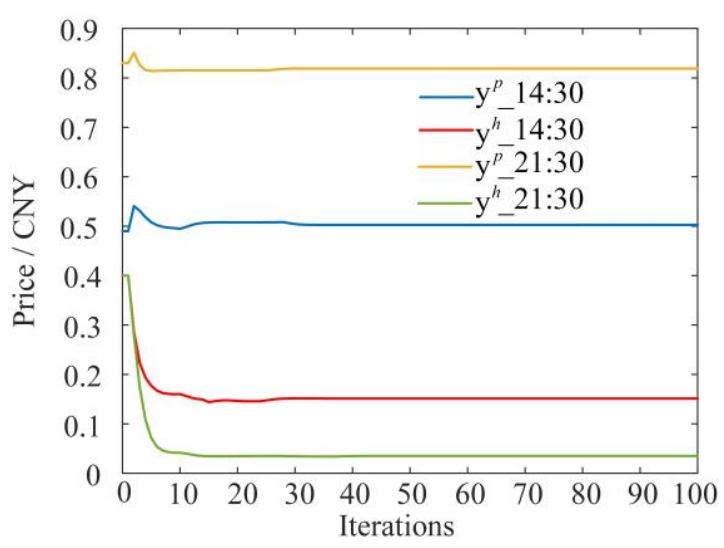

Fig. 12. Iterative process of electrical and thermal prices.

Fig. 12 depicts the iterative process of electrical and thermal prices. The electrical and thermal prices for energy sharing at $14: 30$ are $0.819 \mathrm{CNY} / \mathrm{kWh}$ and $0.151 \mathrm{CNY} / \mathrm{kWh}$, respectively, after achieving convergence. The electrical price is between the selling and buying prices of the utility grid at 14:30. MECM4 prefers to fill electricity demand deficiencies through energy sharing considering the cost of trading with other MECMs or the utility grid and network cost. At 21:30, the electric price is $0.503 \mathrm{CNY}$, which consists of the network cost, and the thermal price is $0.035 \mathrm{CNY}$.

As mentioned in Section IV, the optimization solution of problem (20) can be obtained when the nonconvex constraints are Boolean. This can be referred to in Chapter 9 [14] for more details. The same total operating cost for centralized and distributed scheduling shown in Table $\mathrm{V}$ demonstrates the effectiveness of the ADMM-based decomposition approach. However, we must admit that the global convergence of the proposed energy sharing co-optimization model with binary variables may not be guaranteed. To address this problem, a heuristic approach including relax-round-polish procedures could be adopted to enhance the convergence performance of the ADMM. The interested reader is encouraged to refer to [32] for further details.

\section{CONCLUSION}

This study presents a transactive energy mechanism-supported economic management for multiple MECMs considering uncertainty factors. The scheme includes an IDR strategy at the MECM level and, more importantly, multi-energy sharing of both electricity and heat at the RIES level. Furthermore, an average-based ADMM optimization is adopted to solve the problem of the shared multi-energy supply-demand balance, which is interpreted as a market clearing mechanism from the perspective of transactive energy. Case study results demonstrate that the multi-energy sharing scheme including IDR can take advantage of source flexibility and enrich the operation mode of an RIES. Compared with a case in which all MECMs work in an independent mode, each MECM's cost is reduced in the multi-energy sharing mode, which confirms the benefits for the participating MECMs. In addition, electrical and thermal energy prices are derived by distributed optimization, which provides an effective way to facilitate multi-energy sharing. It can be inferred that with the increasing number of prosumers, the benefits of distributed multi-energy sharing are becoming more prominent.

This work promotes a new solution for the economic and flexible operation of an RIES, and this solution can be extended in multiple directions. For future work, more complex multi-energy sharing including hydrogen energy can be suggested. Considering the differences in dynamic behavior and network constraints of heterogeneous energy systems, the multi-energy sharing model needs to be more refined.

\section{REFERENCES}

[1] Morstyn T , Farrell N , Darby S J , et al, "Using peer-to-peer energy trading platforms to incentivize prosumers to form federated power plants," Nature Energy, vol. 3, no. 2, pp. 94-101, Feb. 2018.

[2] Y. Liu, Y. Li, H. B. Gooi, et al, "Distributed robust energy management of a multi microgrid system in the real-time energy market," IEEE Trans. Ind. Appl., vol. 10, no. 1, pp. 396-406, Jan. 2019

[3]K. Zhou, S Yang, Z. Shao, et al, "Optimal operation of DES/CCHP based regional multi-energy prosumer with demand response," Appl. Energy, vol. 167, pp. 353-365, Apr. 2016.

[4]T. Lu, Z. Wang, Q. Ai, et al, "Interactive model for energy management of clustered microgrids," IEEE Trans. Sustain. Energy, vol. 53, no. 3, pp. 1739-1750, Jan. 2017.

[5]C. Long, J. Wu, Y. Zhou, et al, "Peer-to-peer energy sharing through a two-stage aggregated battery control in a community microgrid," Appl. Energy, vol. 226, pp. 261-276, Sep. 2018.

[6]M.R. Alam, M. St-Hilaire, T. Kunz, "An optimal P2P energy trading model for smart homes in the smart grid," Energy Effic., vol. 10, no. 2, pp, 1-19, Dec. 2017.

[7] K. E. Antoniadou-Plytaria, I. N. Kouveliotis-Lysikatos, P. S. Georgilakis, et $a l$, "Distributed and decentralized voltage control of smart distribution networks: Models, methods, and future research," IEEE Trans. Smart Grid, vol. 8, no. 6, pp. 2999-3008, Nov. 2017.

[8]R. B. Melton, "Gridwise transactive energy framework version 1," Grid-Wise Archit. Council, Richland, WA, USA, Tech. Rep. PNNL-22946, 2015.

[9]D. Gregoratti, J. Matamoros, "Distributed energy trading: The multiple-microgrid case," IEEE Trans. Ind. Electro., vol. 62, no. 4, pp. 2551-2559, Apr. 2015.

[10] T. Morstyn and Malcolm D. McCulloch, "Multiclass energy management for peer-to-peer energy trading driven by prosumer preferences," IEEE Trans. Power Syst., vol. 34, no. 5, pp. 4005-4014, Sep. 2019. 
[11] J. Wang, H. Zhong, J. Qin, et al, "Incentive mechanism for sharing distributed energy resources," J. Mod. Power Syst. Clean Energy, vol. 7, no. 4, pp. 837-850, Jul. 2019.

[12] H. K. Nguyen, A. Khodaei and Z. Han, "A big data scale algorithm for optimal scheduling of integrated microgrids," IEEE Trans. Smart Grid, vol. 9, no. 1, pp. 274-282, Jan. 2018.

[13] J. Hu, G. Yang, K. Kok, et al, "Transactive control: a framework for operating power systems characterized by high penetration of distributed energy resources," J. Mod. Power Syst. Clean Energy, vol. 5, no. 3, pp. 451-464, May. 2017.

[14] S. Boyd, N. Parikh, E. Chu, et al, "Distributed optimization and statistical learning via the alternating direction method of multipliers," Foundations and Trends in Mach. Learning, vol. 3, no. 1, pp. 1-122, 2010.

[15] J. Rivera, C. Goebel, and Hans-Arno Jacobsen, "Distributed convex optimization for electric vehicle aggregators," IEEE Trans. Smart Grid, vol. 8, no. 4, pp. 1852-1863, Jul. 2017.

[16] F. Moret and P. Pinson, "Energy collectives: A community and fairness based approach to future electricity markets," IEEE Trans. Power Syst., vol. 34, no. 5, pp. 3994-4004, Sep. 2019.

[17] W. Huang, N. Zhang, C. Kang, et al,. From demand response to integrated demand response: review and prospect of research and application. Protection and Control of Modern Power Systems, 2019, 4(2): 148-150

[18] L. Ma, N. Liu, J. Zhang, "Real-Time rolling horizon energy management for the energy-hub-coordinated prosumer community from a cooperative perspective," IEEE Trans. Power Syst., vol. 34, no. 2, pp. 1227-1242, Oct. 2018

[19] K. Zhou, S Yang, Z. Shao, et al, "Optimal operation of DES/CCHP based regional multi-energy prosumer with demand response," Appl. Energy, vol. 167, pp. 353-365, Apr. 2016.

[20] J. Yang, A. Botterud, N. Zhang, et al "A cost-sharing approach for decentralized electricity-heat operation with renewables" IEEE Trans. Sustain. Energy, pp. 1-1, Sep. 2019. DOI: 10.1109/TSTE.2019.2943452, [Online].

[21] V.H. Bui, A. Hussain, Y.H. Im, et al, "An internal trading strategy for optimal energy management of combined cooling, heat and power in building microgrids," Appl. Energy, vol. 239, pp. 536-548, Apr. 2019.

[22] A. Ali, N. Liu, and L. He, "Multi-party energy management and economics of integrated energy microgrid with PV/T and combined heat and power system," IET Renew. Power Gener., vol. 13, no. 3, pp. 451-461, Feb. 2019.

[23] N. Liu, J. Wang, L. Wang, "Hybrid energy sharing for multiple microgrids in an integrated heat-electricity energy system," IEEE Trans. Sustain. Energy, vol. 10, no. 3, pp. 1139-1151, Jun. 2019

[24] N. Liu, J. Wang, X. Yu, et al, "Hybrid energy sharing for smart building cluster With CHP System and PV Prosumers: A coalitional game approach," IEEE Access, vol. 6, pp. 34098 - 34108, Jun. 2018

[25] J. E. Contreras-Ocaña, M. R. Sarker, M. A. Ortega-Vazquez, "Decentralized coordination of a building manager and an electric vehicle aggregator," IEEE Trans. Smart Grid, vol. 9, no. 4, pp. 2625-2637, Jul. 2018.

[26] X. Jin, X. Wang, Y. Mu, et al, "Optimal scheduling approach for a combined cooling heating and power building microgrid considering virtual storage system," in IEEE Power and Energy Society General Meeting (PESGM), Boston, USA, 2016, pp. 1-5.

[27] Y. He, B. Venkatesh, L. Guan, et al, "Optimal scheduling for charging and discharging of electric vehicles," IEEE Trans. Smart Grid, vol. 3, no. 3, pp. 1095-1105, Jul. 2012

[28] C. Zhuo, K. Qian, M. Allan, et al, "Modeling of the cost of EV battery wear due to V2G application in power systems," IEEE Trans. Energy Conver., vol. 26, no. 4, pp. 1041-1050, Dec. 2011.

[29] S. Sannigrahi, S. R. Ghatak and P. Acharjee. "Multi-Scenario based Bi-level coordinated planning of active distribution system under uncertain environment," IEEE Trans. Ind. Appl, vol. 56, no. 1, pp. 850-863, Jan-Feb. 2020

[30] M. Juelsgaard, P. Andersen, R. Wisniewski, "Distribution loss reduction by household consumption coordination in smart grids," IEEE Trans. Smart Grid, vol. 5, no. 4, pp. 2133-2144, Jun. 2014.

[31] "Gurobi optimizer reference manual," [online] Available: http://www.gurobi.com
[32] C. He, L. Wu, T. Liu, et al, "Robust co-optimization scheduling of electricity and natural gas systems via ADMM," IEEE Trans. Sustain. Energy, vol. 8, no. 2, pp. 658-670, Apr. 2017.

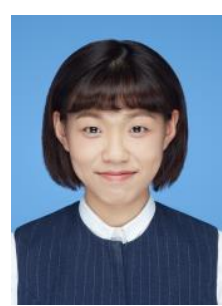

Zhao Yang received the B.S. degree from Southwest Jiaotong University, Chengdu, China, in 2018. She is currently working toward the M.S. degree in the School of Electrical and Electronic Engineering, North China Electric Power University, Beijing, China.

Her research interests include transactive energy and the optimal operation of integrated energy system.

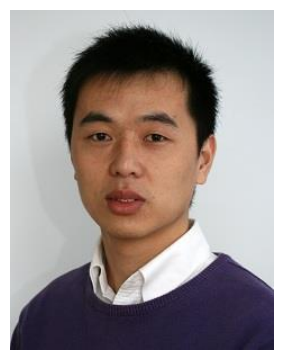

Junjie. Hu (M14) received his M.Sc. degree in control theory and control engineering from Tongji University, China, in 2010, and the Ph.D. degree in electrical engineering from the Technical University of Denmark, Denmark, in 2014. He was a postdoc researcher with the Department of Electrical Engineering, Technical Unversity of Denmark. He is currently an Associate Professor with School of Electrical and Electronic Engineering, North China Electric Power University. His current research interests include distributed energy resources energy management, transactive energy, prosumers energy management, multi-energy system optimization.

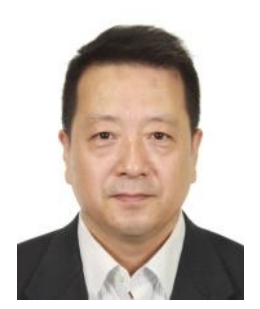

Xin Ai (M'17) received the B.S. degree from the Nanjing Institute of Technology, Nanjing, China (now Southeast University); the M.S. degree from the China Electric Power Research Institute, Beijing, China; and the Ph.D. degree from North China Electric Power University (NCEPU), Beijing, in 1985, 1988, and 1999, respectively, all in electrical engineering. He was a Senior Research Scholar with Brunel University, London, U.K., in 2003. He was the Director of the Institute of Power Systems, NCEPU, where he was engaged in research and teaching on power system and automation. He is currently a Professor and a Doctoral Tutor with the School of Electrical and Electronic Engineering, NCEPU. His current research interests include power system analysis and control, and transactive energy.

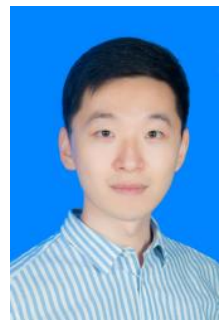

Jiechen Wu received the B.S. degree from Northeast Electric Power University, Jilin, China, in 2014. He received the $\mathrm{Ph} . \mathrm{D}$. degrees in electric engineering from North China Electric Power University, Beijing, China, in 2020. Currently, he is working in the State Grid Economic and Technological Research Institute Co., Ltd., Changping District, Beijing, China.

His research interests include transactive energy and energy Internet.

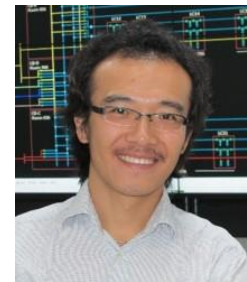

Guangya Yang (SM'14) received the B.E., M.E., and $\mathrm{Ph} . \mathrm{D}$. degrees in electric power system in 2002, 2005, and 2008. He is currently an Associate Professor with the Center for Electric Power and Energy, Department of Electrical Engineering, Technical University of Denmark, Kongens Lyngby, Denmark. Since 2009, he has been with the Technical University of Denmark as a Postdoctoral Researcher and has been leading several industrial collaborative projects in Denmark in the field of monitoring, operation, and protection of renewable energy systems. His research interests include renewable energy integration, smart grids, and cyber-physical energy systems. 